\title{
Smoothly Waning, Symmetrically Expanding, Cavity Pressure Loads in Earth Materials
}

\author{
Thomas G. Priddy
}

\section{Prepared by}

Sandia National Laboratories

Albuquerque, New Mexico 87185 and Livermore, California 94550

for the United States Department of Energy

under Contract DE-AC04-76DP00789 
Issued by Sandia National Laboratories, operated for the United States Department of Energy by Sandia Corporation.

NOTICE: This report was prepared as an account of work sponsored by an agency of the United States Government. Neither the United States Government nor any agency thereof, nor any of their employees, nor any of their contractors, subcontractors, or their employees, makes any warranty, express or implied, or assumes any legal liability or responsibility for the accuracy, completeness, or usefulness of any information, apparatus, product, or process disclosed, or represents that its use would not infringe privately owned rights. Reference herein to any specific commercial product, process, or rights. Reference herein to any specific commercial product, process, or
service by trade name, trademark, manufacturer, or otherwise, does not necessarily constitute or imply its endorsement, recommendation, or favoring by the United States Government, any agency thereof or any of their contractors or subcontractors. The views and opinions expressed herein do not necessarily state or reflect those of the United States Government, any agency thereof or any of their contractors or subcontractors.

Printed in the United States of America Available from

National Technical Information Service

U.S. Department of Commerce

5285 Port Royal Road

Springfield, VA 22161

NTIS price codes

Printed copy: $\$ 8.00$

Microfiche copy: A01 
$\mathrm{UC}-2$

SAND $81-2054$

SMOOTHLY WANING, SYMMETRICALLY EXPANDING, CAVITY PRESSURE LOADS IN EARTH MATERIALS

by

T. G. Priddy

Applied Mechanics Division II 5522

Sandia National Laboratories

Albuquerque, NM 87185

\section{Abstract}

An approximate solution for the pressure loading required to expand a spherical cavity in rock and soil targets is derived. A dual characterization of fracture and flow material model is used. Frictional resistance to flow is also included. Non-linear volumetric strain hardening is modeled with bi-linear curves and unloading is assumed to be non-dilative. Applications of this solution form to the prediction of pressure loading on slender, convex-nosed earth penetrators and on spherically-nosed water entry vehicles are discussed. 


\section{Table of Contents}

\section{Abstract}

Page

Introduction

Penetrator Nose Shape, Penetration Speed, Cavity

Models and Material Response Zones 7

Expanding Cavity Problem Statement 19

Stress Relations for Spherical Expansion 21

Material Model

Assumed Particle Velocity Distribution

Within Wake of Crush Front 28

Crush Front Dynamics

Low Speed Conditions 33

Intermediate Speed Zone 36

High Speed Zone 39

Equation of Motion 42

Slow Speed Penetration Cavity Solution, Figure 3

Intermediate Speed Range, Figure 4

High Speed Penetration, Figure $5 \quad 49$

Preliminary Applications $\quad 54$

Case I. Water Entry 55

Case II. Antelope Tuff/DGU12 Test 61

Case III. Mount Helen Welded Tuff 64

$\begin{array}{ll}\text { Conclusions } & 67\end{array}$

Nomenclature 68

Acknowledgements $\quad 72$

List of References: $\quad 73$ 
Introduction

Symmetrically expanding cavity solution forms are useful in predicting the pressure loading on deeply penetrating projectiles[1-8]. In these applications, the penetrator's shape and motion determine the size, rate of expansion and the acceleration of the symmetrical cavity. The pressure required to drive this cavity is then derived and used to approximate the loading on the penetrator.

In the PACE code[1] these calculations are performed at incremental time steps and side loads (asymmetric pressure distributions) along with axial loads are estimated for nearly straight trajectories. The rigid body equations of motion for a penetrator are then numerically integrated forward in time throughout a complete penetration event. The PACE code thereby provides an approximate solution to the three-dimensional response of a penetrator.

The spherical cavity model[2] which is used in the PACE code is based on a locking, elastic-plastic, shear strain hardening material model. Various forms of this generally stated material model, various cavity shapes (cylindrical and spherical) and solution methodologies are also found in References $3,4,5$ and 6 . As illustrated in these references, these approximate techniques have produced good correlations with more detailed numerical calculations and/or with experimentally measured data. 
The PACE code is currently being replaced with a new, more flexible and modular code: GNOME. The objective is to provide a broader coverage of earth material models and approximate solutions. An applicable cavity solution form is sought in this report which better models the physical phenomena associated with the penetration of rock targets with convex nosed penetrators. Specifically, the phenomenon of separated flow as a function of unconfined shear strength (for target materials) penetrator velocity, and ogive nose shape is particularly important to both axial and side load predictions as it determines the existence or absence of frictional drag on cylindrical body sections. Additionally, post fracture modeling of rock materials as plastic behavior without any loss in shear strength would be intuitively unsatisfying. The cavity pressure response to widely varying velocities is desired since loads data taken from relatively slow speed development tests must be scaled upward for some high speed applications. A properly tailored solution for a spherical cavity model is also desired for applications to doubly curved (both blunt as in the case of water entry bodies and slender as in the case of earth penetrators) nose shapes. Finally, a closed form cavity pressure equation is demanded for inexpensive computations since the pressure at many points on the body must be quantified and numerically integrated at a large number of incremental time steps to predict the desired three dimensional response of a penetrator through the interesting parts of relatively long trajectories. 
This report consists of a number of sections which were written to describe the wide ranging concepts used in the study. The next section is an extension of this introduction where the application of simple cavity expansion models to penetrator loads is discussed. A combination of non-linear material behavior, penetrator nose shape and penetration velocity intuitively lead to a set of three zones of target material compaction. A short section then follows where the spherical cavity problem statement is concisely stated. Since new features are introduced for the material response, a separate section is devoted to the material models. Approximations for particle velocity and outer radius stress states are essential to the solution technique and separate sections are therefore provided for each. These are followed by solution of the equation of motion. Preliminary applications and conclusions are then discussed. Since a large number of symbols are used in this development, a nomenclature listing is provided at the end of the report. For reading purposes, symbols are also defined as they are introduced. 
Penetrator Nose Shape, Penetration Speed, Cavity Models and Material Response Zones

A conceptual view of the response of earth material about the nose of a penetrating projectile is discussed in this section. Figure $1-a$ is an illustration of geometrical details, normal velocity and pressure terms for a conical and an ogival nosed penetrator. Both cylindrical and spherical cavity expansion approximations have been used to model the penetrator loads problem. In the cylindrical cavity model, a slice out of a uniformly expanding long cylinder is driven radially outward by an internal boundary velocity of $v_{n} / \cos \phi$. In the spherical model, a tangent sphere is expanded at a velocity of $v_{n}$, where $v_{n}$ is the normal component of the velocity of the penetrator nose. With either model, an internal cavity is formed in the medium by the advancing projectile body and during the period where the body and the soil are interacting; the size, velocity and acceleration of these cavities are determined by the projectile shape and its motion.

Figure $1-b$ is a plot of the cavity velocity distributions for the two nose shapes penetrating at slightly decreasing penetrator velocity, $V$. Following a step increase in velocity at the projectile nose tip, the cavity motion is smooth in time and the cavity velocity $v_{n}$ is "waning" (the acceleration of the cavity is smoothly negative) for both convex and conical nosed 


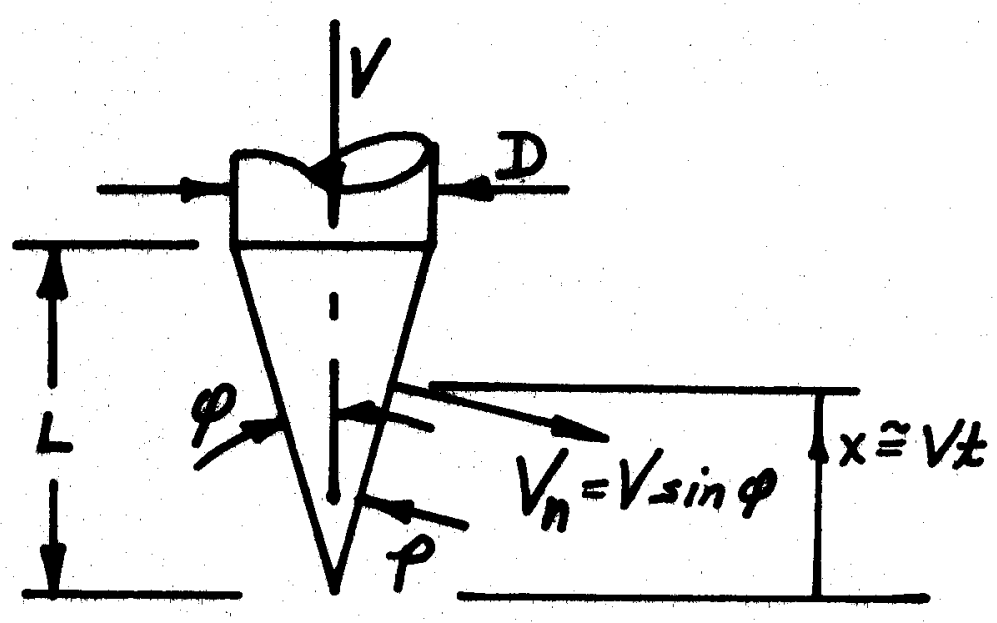

Conical Nose

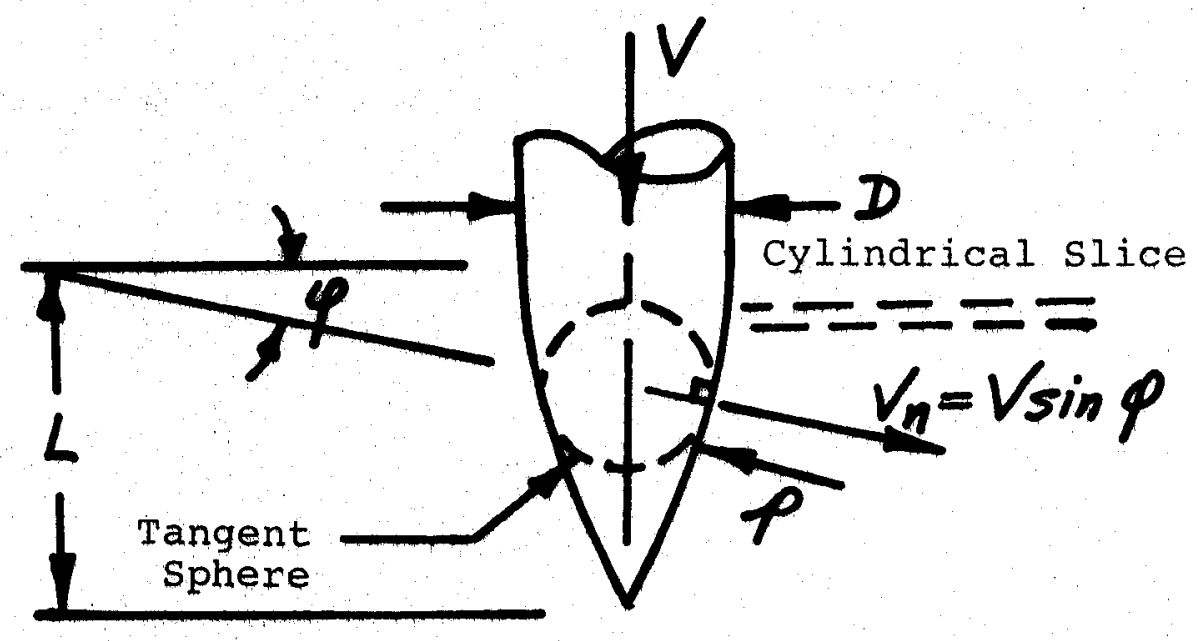

Ogival (Convex) Nose

Figure (1-a)

Nose Shapes

8 


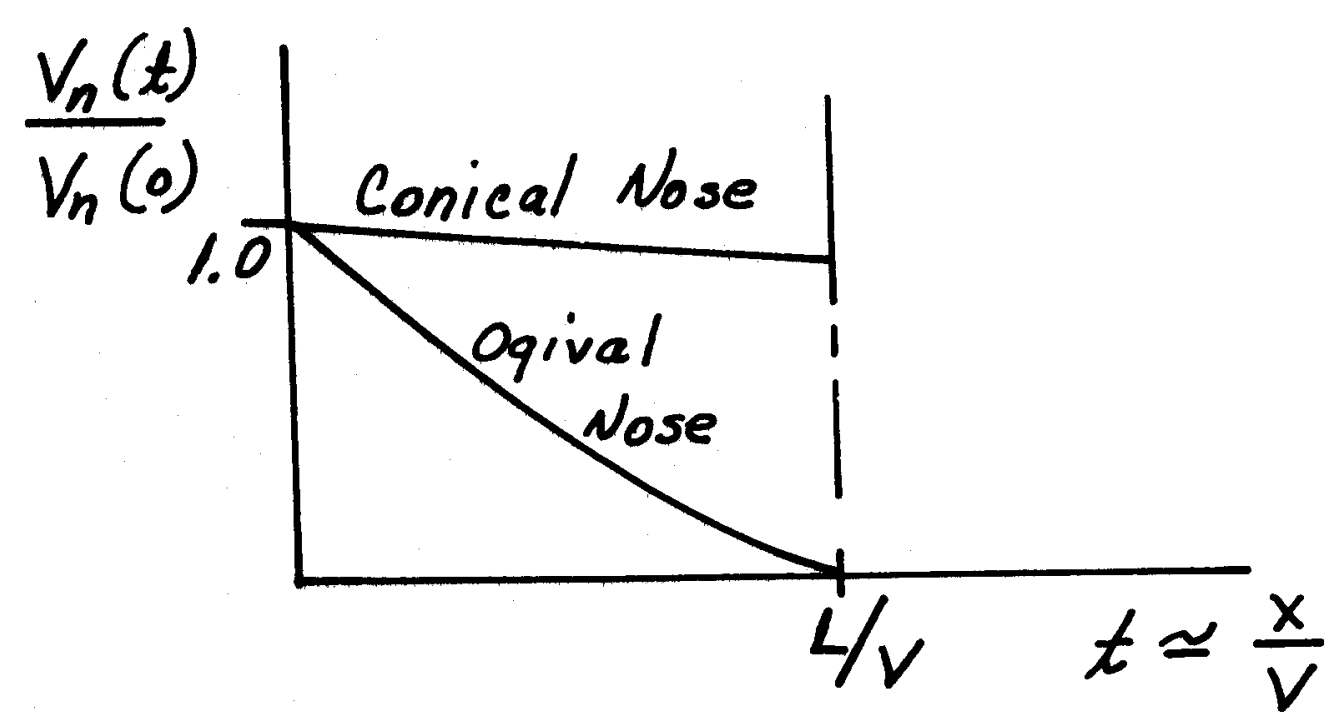

Figure $(1-b)$
Cavity Velocity Distributions

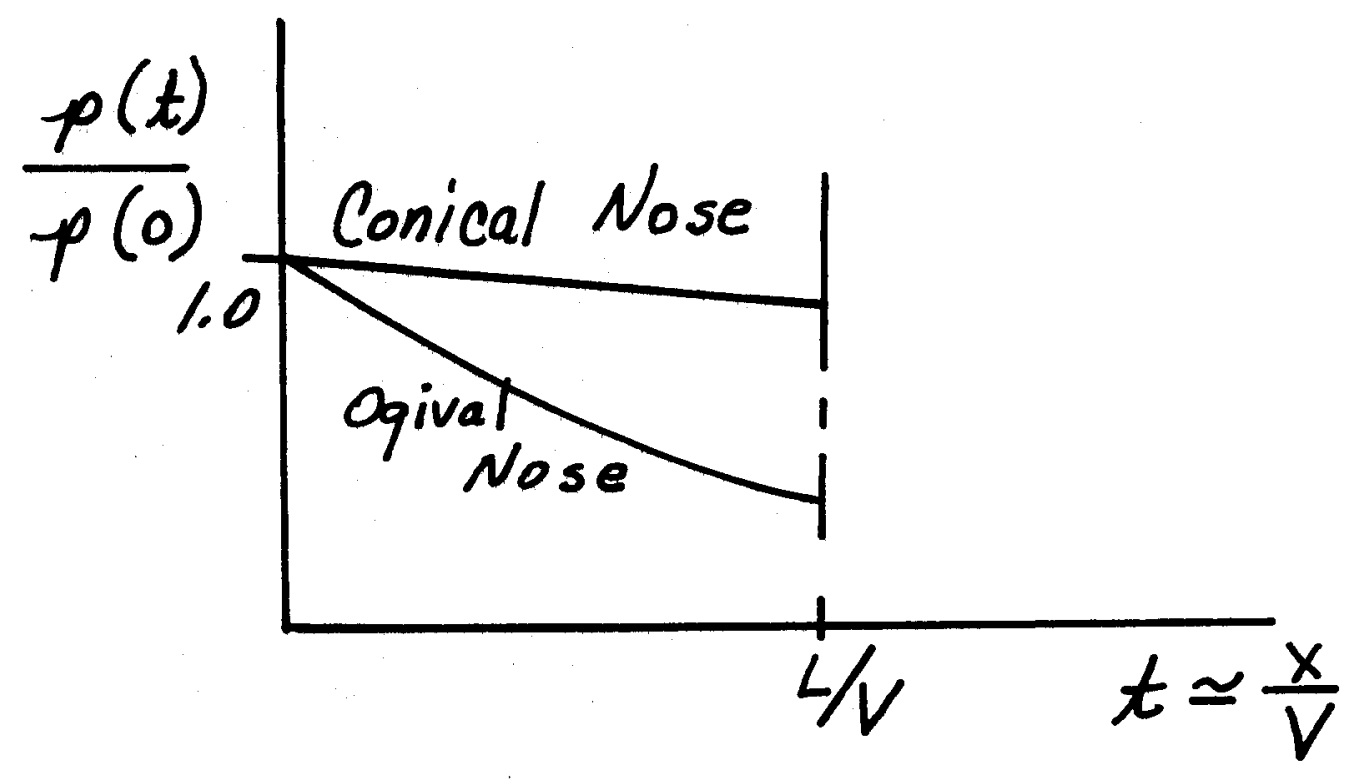

Figure $(1-c)$
Cavity Pressure Distributions

9 
penetrators which are undergoing negative acceleration. For conical nosed penetrators penetrating at constant velocity, the cavity velocity $V_{n}$ is constant.

Figure $1-c$ is an illustration of expected pressure distributions on the two different nose shapes. The time rate of change of pressure on a material element which slides along the convex penetrator nose is negative and the material is "unloading" hydrostatically. "Permanent set" in earth targets is evidenced by a residual hole which persists after a projectile has passed through the medium. Cavity solutions discussed in this report are therefore developed for use in the prediction of pressure loads on smoothly convex or conical shaped projectiles which are slowing down, and as they pass through earth materials.

Characteristically, there are several earth material types. In situ properties vary within each type and with various conditions such as water content. Because of this ever present variability and uncertainty in an untested target site, exact (deterministic) solutions to the physical problem are unattainable in general. Simplistic earth material models which are used for approximate solutions may demand more data themselves than can be provided for the generally stated target. Regardless, a dynamic solution to the rapid expansion of a hole in the ground demands that the material be mathematically modeled and for acceptably accurate approximations, basic 
(first order important) response characteristics of the material must be described and quantified. A material model is presented later in this report but in order to tie the penetrator velocity and dynamics to the material model and to the cavity solution needs, the pressure vs. volumetric strain curve is shown in figure 2 .

Three zones of material response are illustrated in Figures $3,4,5$ and these correspond to various penetrator velocities. Figure 3 is an illustration of slow speed penetration into competent rock. Material which enters the fractured and compacted zone must be crushed by a radial stress (at the outer boundary, R) which is sufficient to fracture the material in a quasi-static rate of loading. Wave front stresses which propagate outward from the internal disturbance are too weak by definition in this speed regime to crush the rock. Conditions necessary for the existence of this type of response are therefore material strength and cavity velocity dependent. The critical velocity (maximum allowed for this type of expansion) depends on the cohesive strength of the earth material in question. By definition, material outside this inner zone is stressed elastically. 


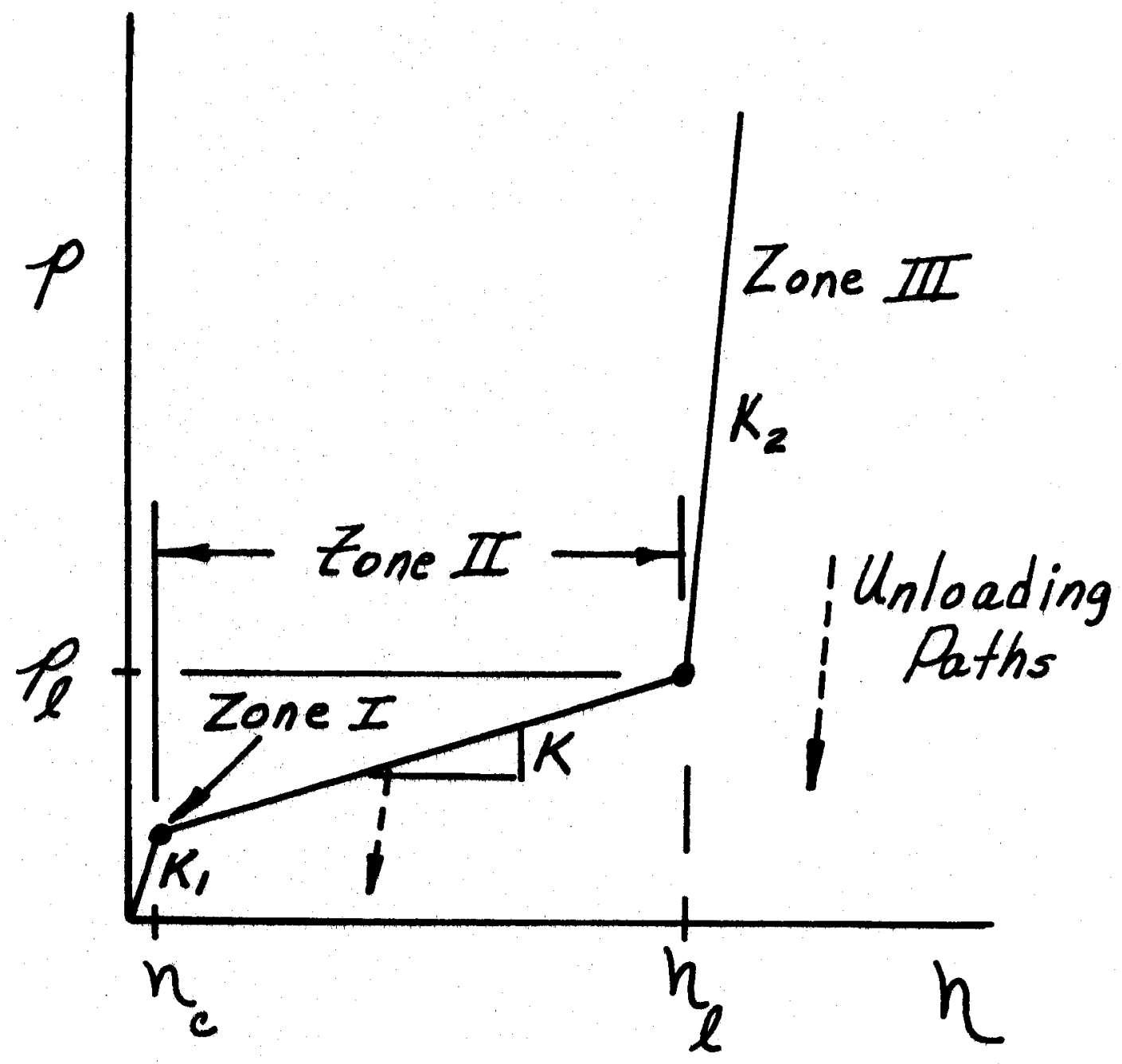

Figure 2

Pressure-Volume Strain

With Approximate Unloading Paths for

Various Zones Illustrated in Figures $(3,4$, and 5 ) 


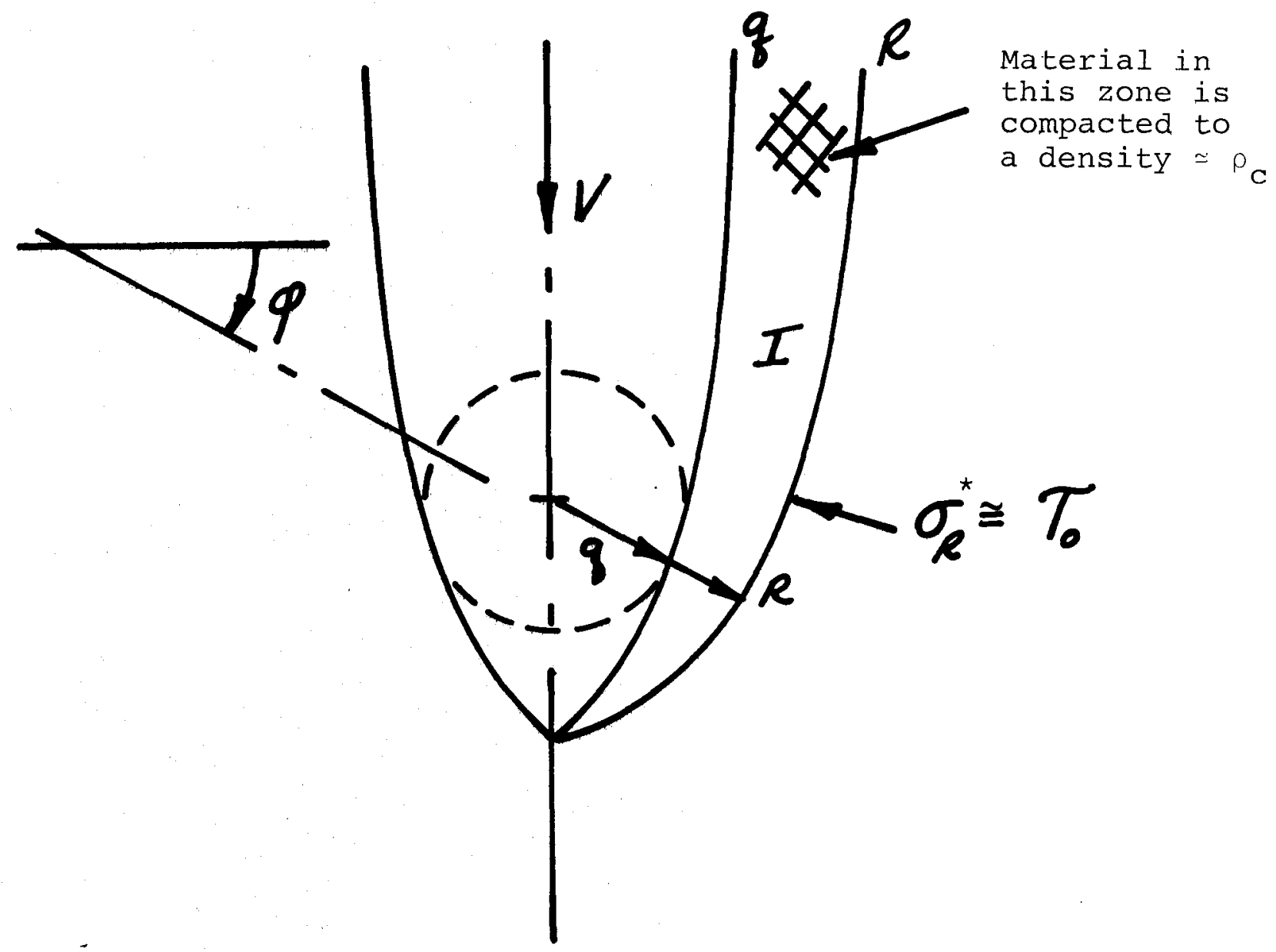

Figure 3

Slow Speed Penetration into Competent So 17 and Rock Targets

*Expression for $\sigma_{R}$ shown on this figure as well as those shown on Figures 4 and 5 are developed later in report. 
Figure 4 is an illustration of response to intermediate velocities. The material near the penetrator nose tip is loaded sufficiently fast to shock-crush the rock but not strongly enough to "bottom out" the material in a hydrostatic sense; i.e., the pressure at the wave front is less than $p_{l}$ shown in Figure 2. Since the stress just behind this front decays with negatively accelerating expansions (convex nosed penetrators) the advancing crush front conditions switch to that described in Figure 3 at some time in the expansion. Continued expansion is resisted by the material's strength, the acceleration of the accumulated zone of crushed rock, and the attendant frictional shear resistance to continued flow of the material in these zones.

Figure 5 is an illustration of behavior to be expected under high speed expansions. The material near the tip is first shock loaded to pressures which exceed $p_{l}$ shown in Figure 2. This zone advances until the material at the front is loaded to just $p_{\ell}$. Continued response at the wave front is the same as that of Zone II in Figure 4 which subsequently decays at a later time to the quasi-static condition.

As illustrated, there is a strong relationship between material properties and expansion rate in distinguishing between low, intermediate, and high speed response.

Expanding cavity solutions which are useful for predicting pressure loading on smoothly convex or conical nosed penetrators must, in general, take into account the following: 


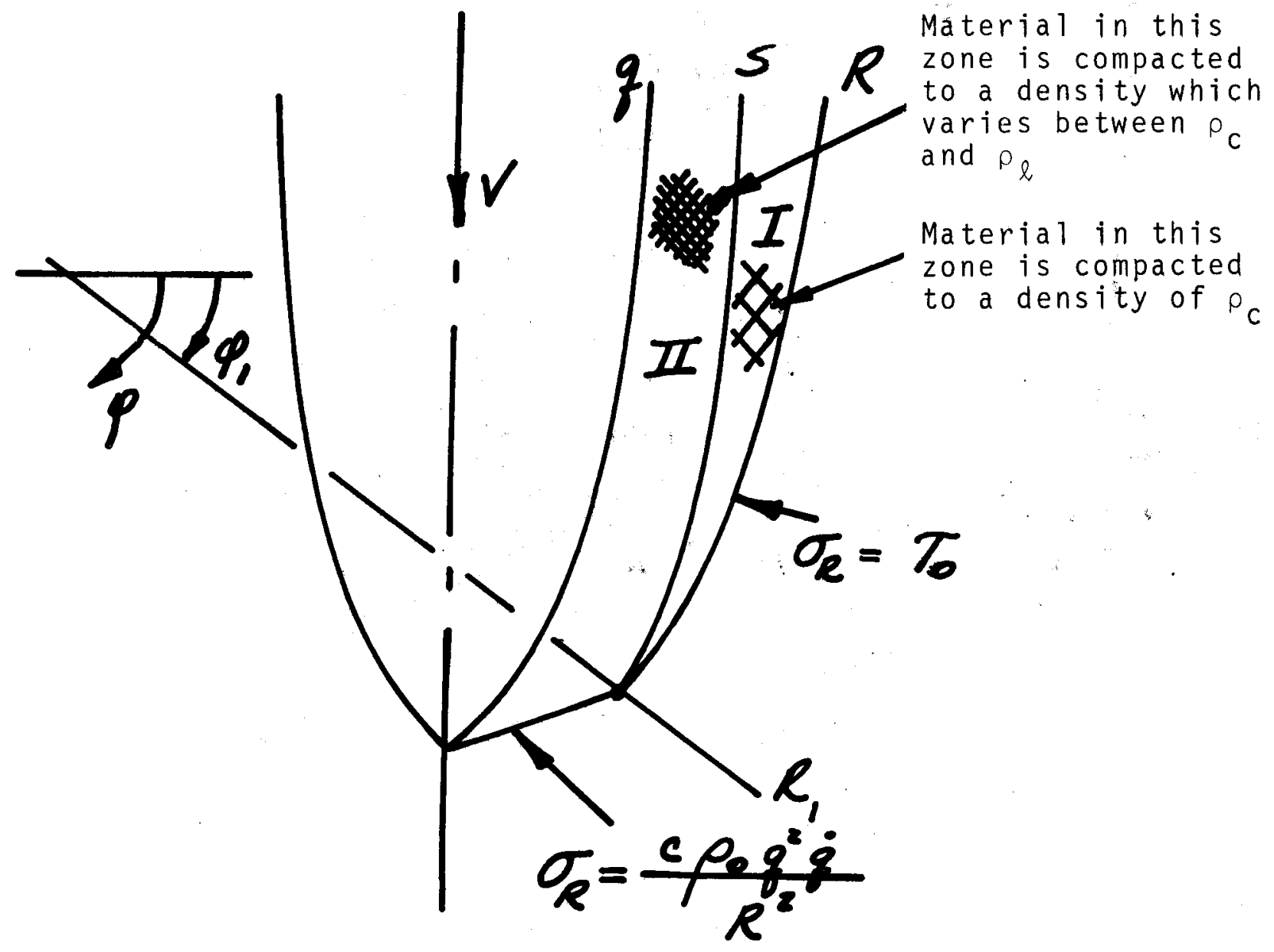

Figure 4

Intermediate Speed Penetration into

Competent Soil and Rock Targets 


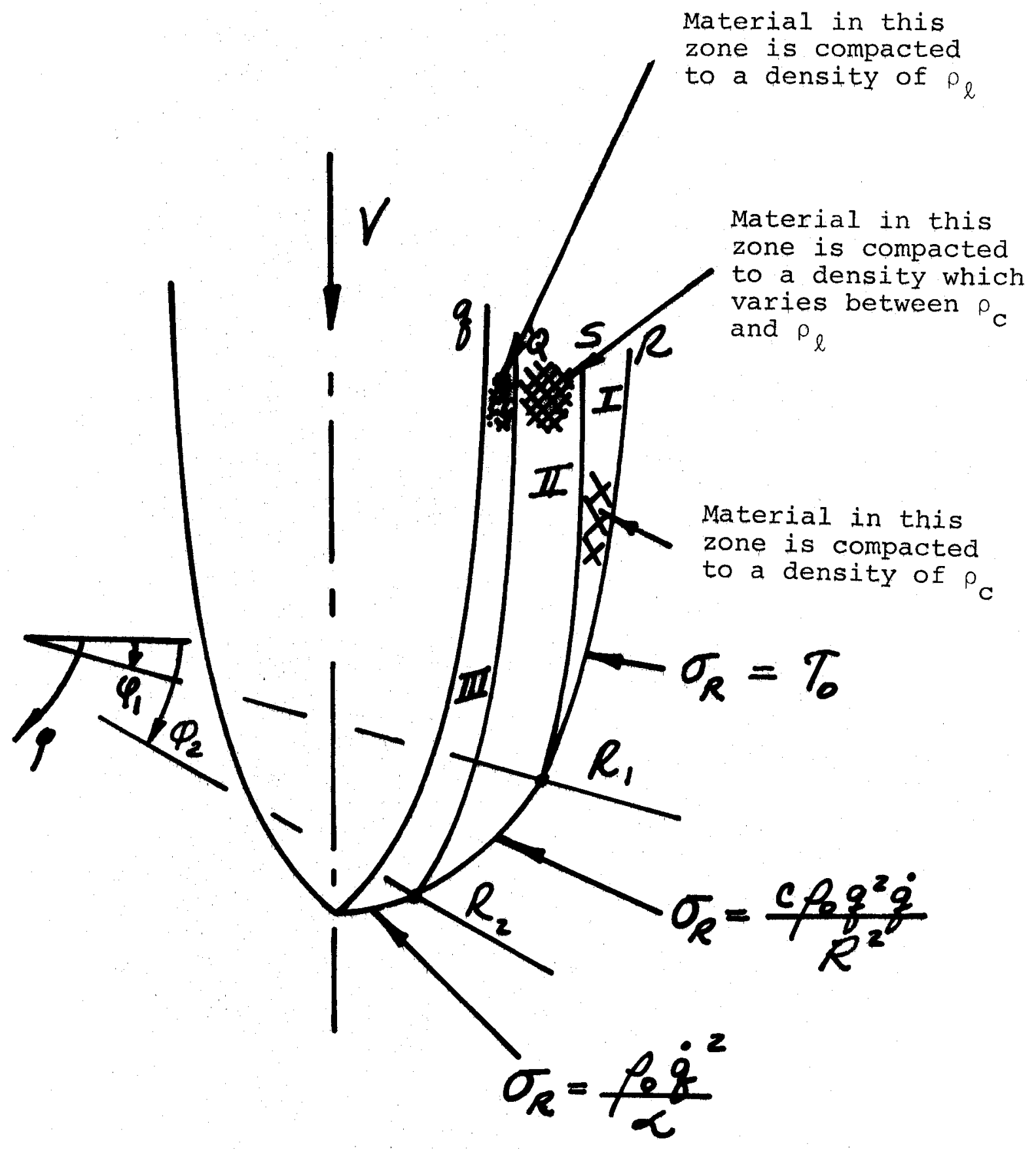

Figure 5

High Speed Penetration into Competent Soil and Rock Targets 
1. Peak pressure occurs at the nose tip (zero radius in the symmetrical cavity models) and the crushed and compacted material which bears against the penetrator surface undergoes unloading for convex nose shapes and remains at nearly a constant pressure for conical shapes. Material which lies within the wake behind the leading crush front may continue to be compacted at a relatively slow rate (as in the case of constant velocity, conical nose penetration, Ref. 7) or it may experience unloading for waning expansions. Rapid compaction of the earth material may occur at the leading front. The unloading $p-\eta$ relationship is important in some cases for material within the crushed and compacted zones. Loading-up of this material depends on the usually measured $p-\eta$ curve which is important in all cases at the leading front.

2. Provision for the possibility that solid like compaction will occur with "high speed" penetration is needed, i.e., with a piece-wise linear $p-n$ curve as selected here, three zones of compaction within the wake may occur.

3. Shear strain in zones near the penetrator body (inner cavity) is finite and continues to grow with the expansion. This shear strain in zones near the cavity greatly exceeds the elastic limit of earth materials. Shear stress (resistance to flow) in these zones of crushed rock is primarily derived from internal friction, i.e., the initial cohesive strength of rock, for example, is destroyed by fracturing at the leading front. 4. Smooth kinematic forcing at the inner cavity surface together with smooth progression of the leading crush-front in 
a homogeneous material model indicates a smoothly declining pressure distribution along the nose of the penetrator. Vibratory motion witnin the wake zones is possible but the disturbances or non-homogeneities necessary to excite and sustain this type of response are omitted in this report. The objective of this development is to describe approximate solutions to spherically expanding cavities which are suitable for predicting the pressure loading on deeply penetrating projectiles. A material model is used which will approximately and simply describe measured behavior of several earth material types. The resulting closed form solutions are useful in inexpensive computations of the response of a body via full penetration trajectory codes. Asymmetric perturbations[1] to the cavity velocity and acceleration may be employed to predict lateral loading for nearly straight penetration events. 


\section{Expanding Cavity Problem Statement}

With reference to Figure 6 , consider an expanding spherical cavity in rock or soil. At the inner boundary, $r=q$; the velocity and acceleration are given by $\dot{q}$ and $\ddot{q}$ and these along with q are prescribed. These are assumed to be smooth in time following a step increase in $\dot{q}$ at zero time. The velocity, $\dot{q}$, is always positive and the acceleration, $\ddot{q}$, is zero or negative for the period of interest. At zero time, $q$ is zero and the medium is assumed to be stress free and to have a density o. A differential element of fractured or crushed material, at a later instant in time, positioned at a radial distance $r$, is compacted to a density o by the compressive stress components $\left(\sigma_{r}, \sigma_{\phi}, \sigma_{\theta}\right)$ where $\sigma_{\theta}=\sigma_{\phi}$. The outer boundary, $r=R$, is at the moving front which defines the boundary between elastically stressed material and the "plastically" compacted zone. The elastic stress at this interface is assumed to be negligible except in low velocity penetration and/or late time where the static crush strength controls. The crushed or compacted region may be further divided into speed and material dependent response zones. Radial stress at the inner boundary, $\sigma_{q}$, is the unknown to be determined. 


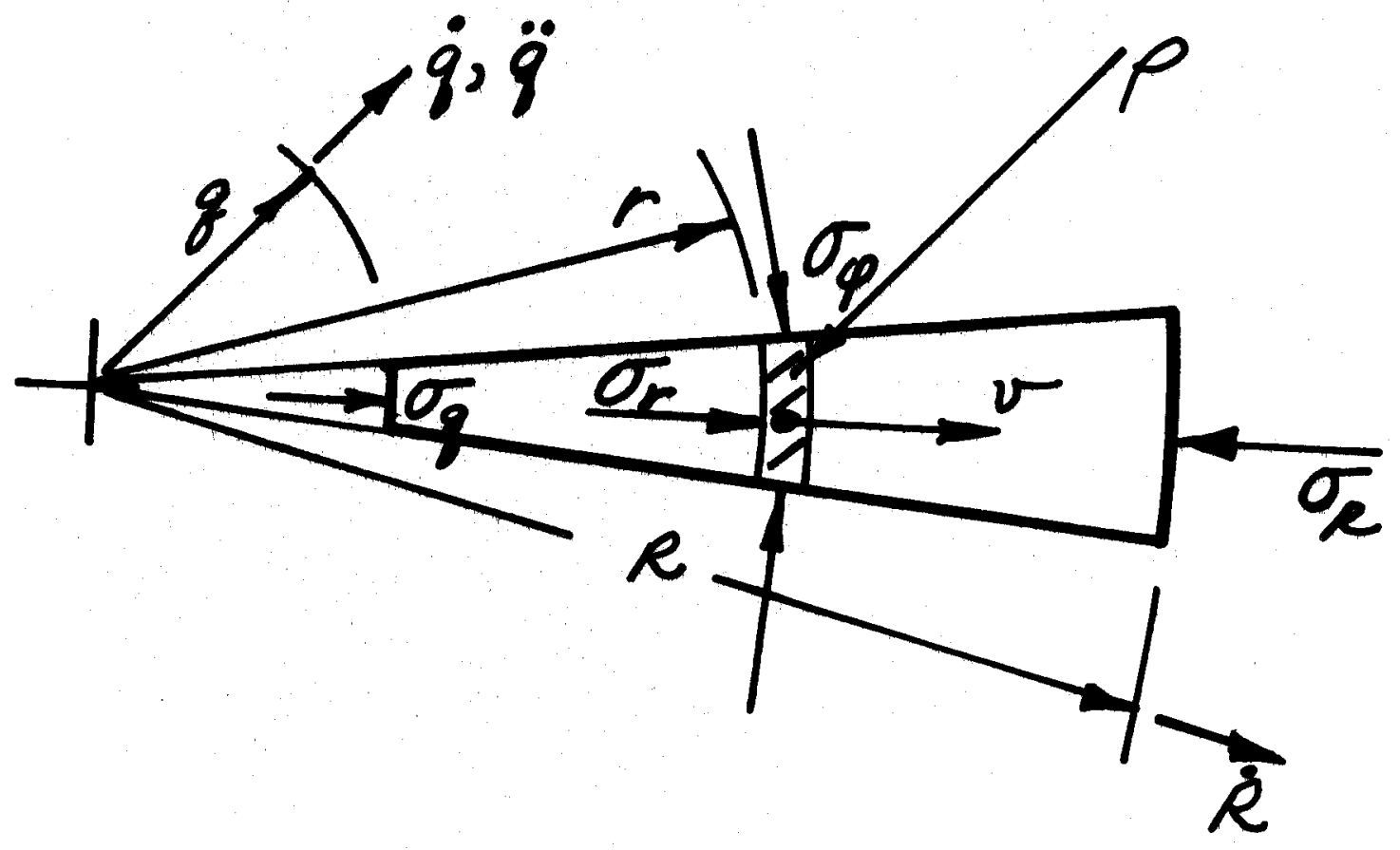

Figure 6

Expanding Spherical Cavity Problem 
Stress Relations for Spherical Expansion

Hydrostatic pressure and stress difference (shear) are defined with:

$$
\begin{aligned}
& p=\frac{1}{3}\left(\sigma_{r}+2 \sigma_{\phi}\right) \\
& \tau=\sigma_{r}-\sigma_{\phi}
\end{aligned}
$$

Inversely:

$$
\begin{aligned}
& \sigma_{r}=p+\frac{2 \tau}{3} \\
& \sigma_{\phi}=p-\frac{\tau}{3}
\end{aligned}
$$

21 
Material Model

Basic coverage of the response of homogeneous regions of in situ geologic materials (to a simple first approximation) is modeled with Figures 7,8 and 9. For symmetrical expansions and when the stress state is separated into dilatational and deviatoric components, two components of stress (pressure and shear) must be defined.

Figure 7 is a piecewise linear model for pressure versus volumetric strain. In this model, $k_{1}$ as shown in Figure 2 is assumed to be approximately equal to $K$. Several materials may be approximately characterized with this model. For examples: the idealized locking model would have $k=0$ and $k_{2}=\infty$; while a simple linear approximation would place $p_{\ell}$ at infinity. In general, $K$ (the volumetric stiffness) is not zero and some kind of hardening is needed for higher pressure loads. Effects of water content can be treated as it affects the slopes of the linear segments, the in situ density and the distended volume range $\left(n_{l}\right)$. Unloading paths are important in the waning cavity expansion problem. Unloadings of compacted, fractured and highly distorted soil and rock materials are not expected to follow the same path as measured in loading up tests. More steeply unloading slopes are expected with some degree of reconsolidation and/or "locking." For convenience, the unloading slope is assumed to be nearly $k_{2}$ and $k_{2}$ is 


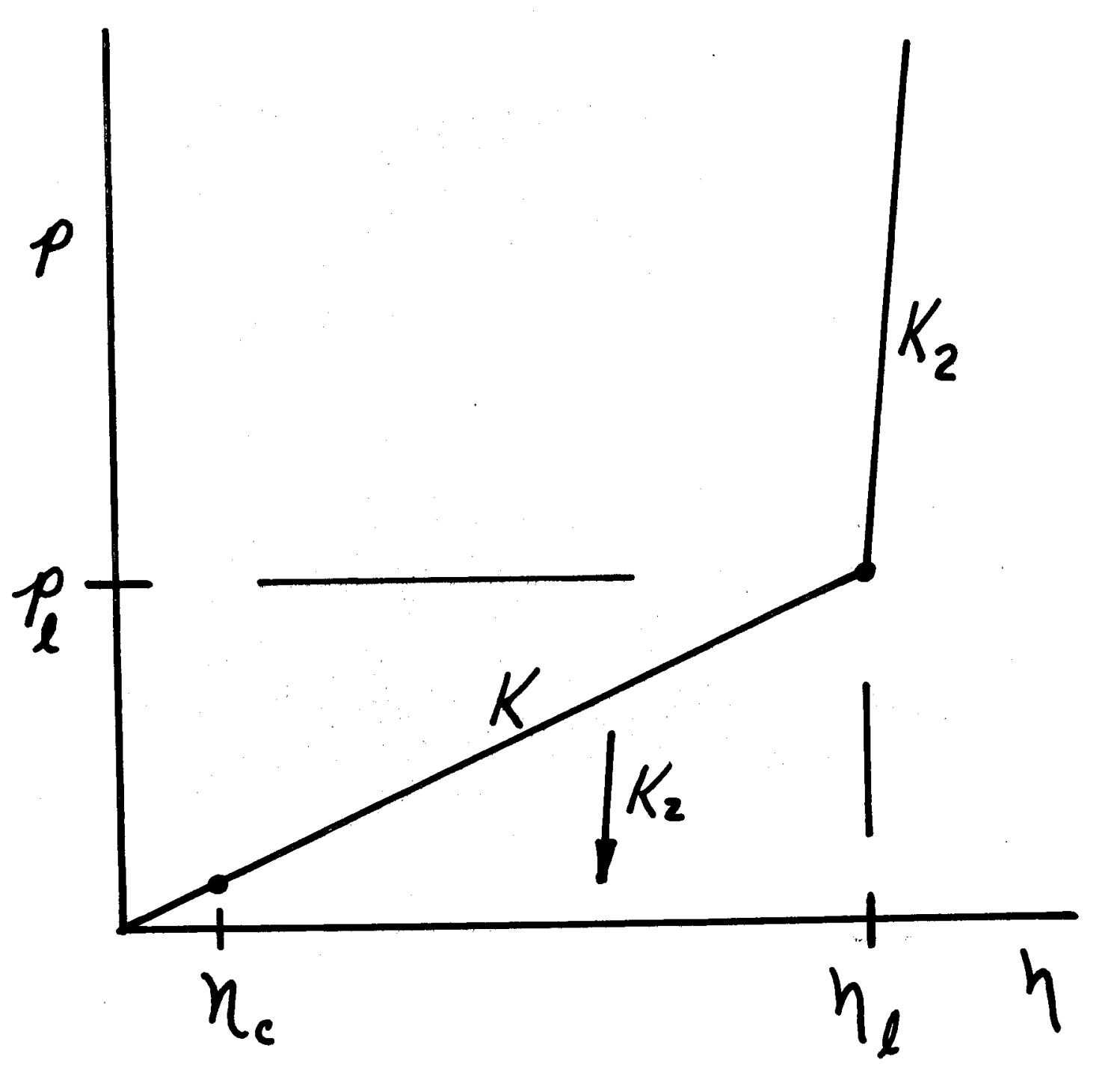




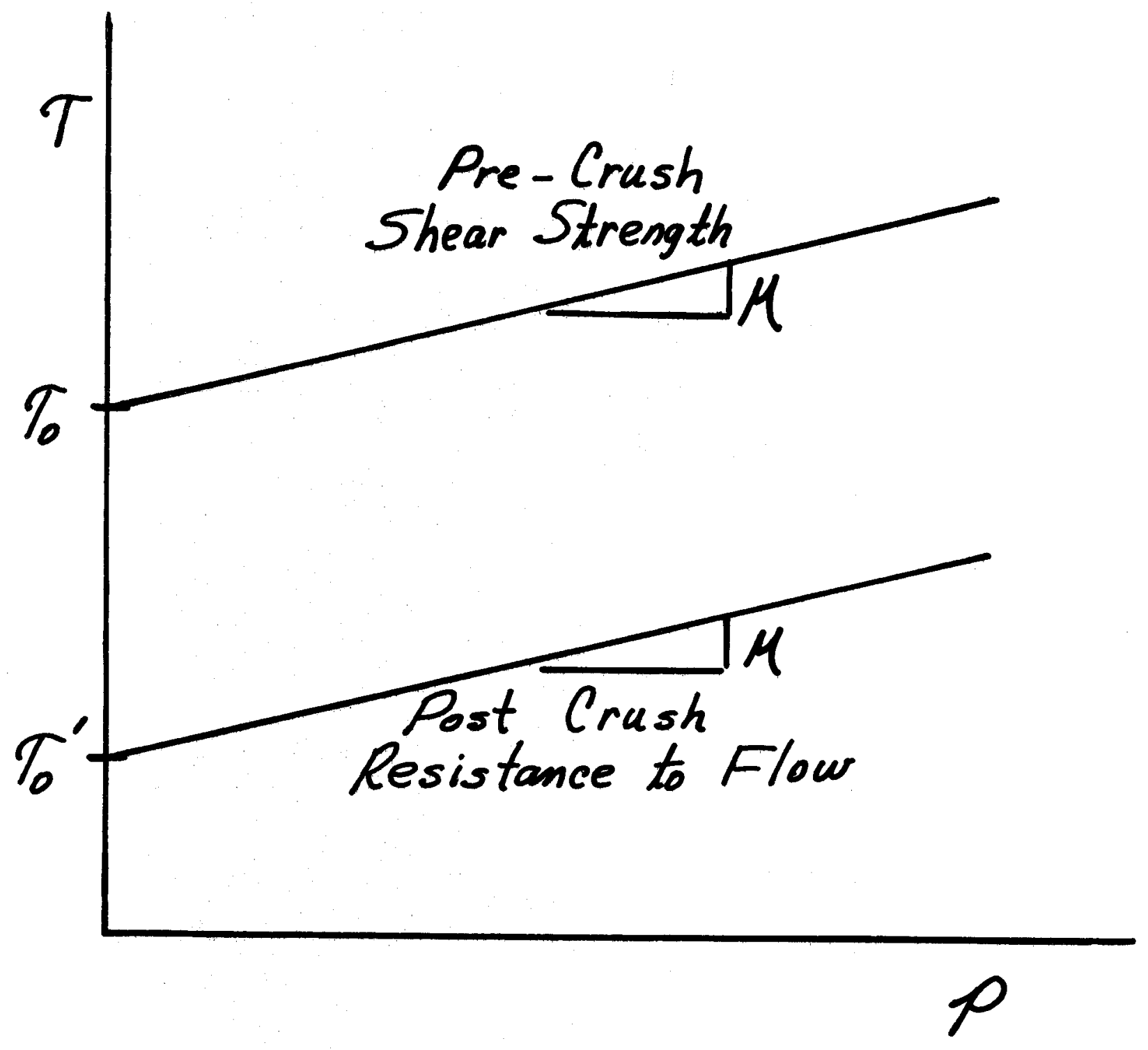

Figure 8

Fracture and Flow Strength Models 


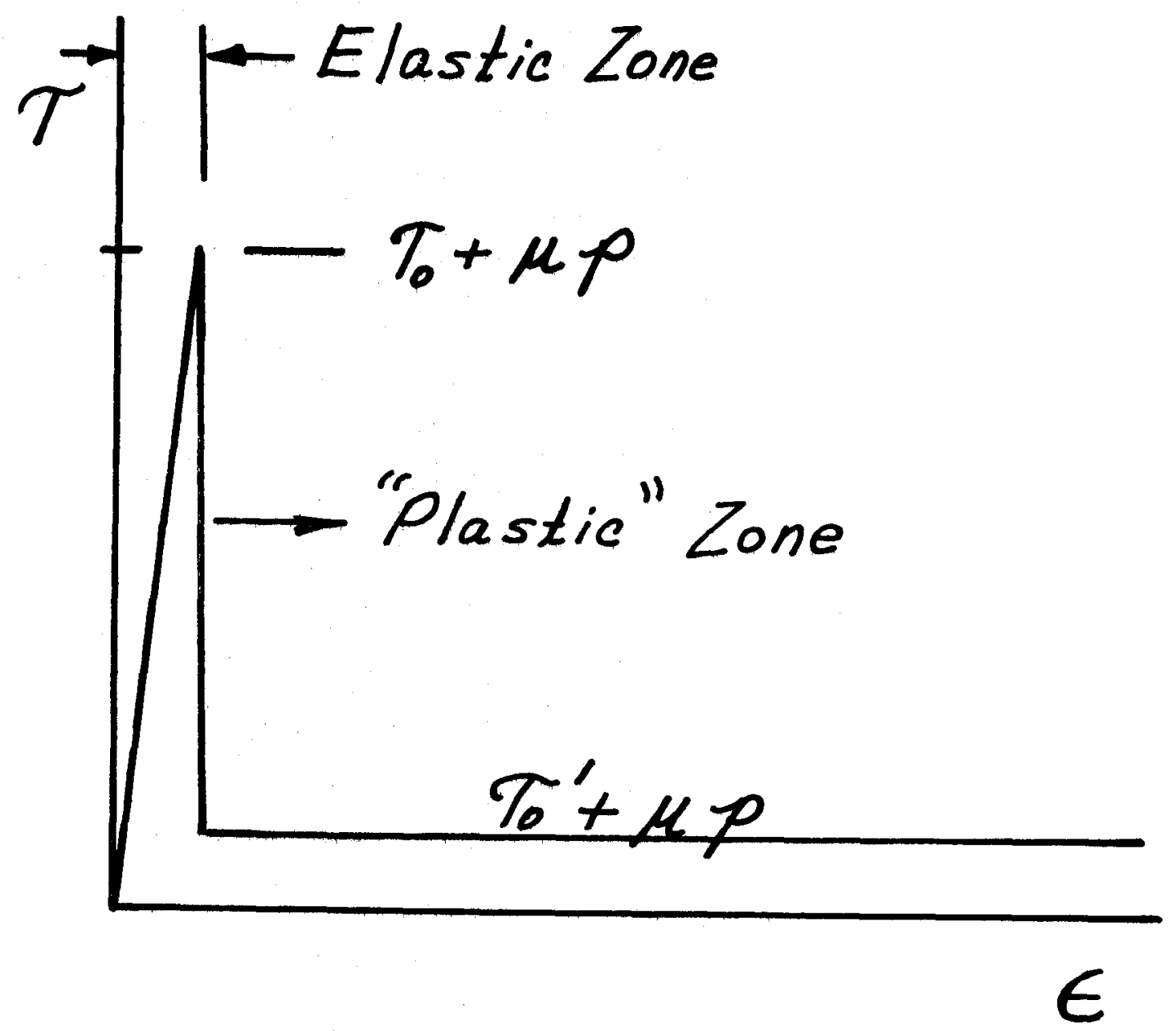

Figure 9

Shear Stress-Shear Strain Model 
assumed to be nearly vertical in our problem. Yarrington (4) used a generalized locking on unloading model of this kind in his cylindrically expanding cavity techniques.

Shear resistance to finite deviatoric strain and continuously outward flow conditions is illustrated with Figures 8 and 9 and modeled with Equation 5 . Depending on the soil or rock type and the moisture content, shear resistance to flow depends on various factors. A constant or residual cohesive like resistance, $\tau_{0}^{\prime}$, may be found in some clays while frictional resistance is found in wet and dry sands.

A fracture or cohesive strength is available in competent rock targets. This brittle strength must be exceeded at the advancing front of the inner zones of the expansion but once broken this strength is lost or reduced in magnitude and the shear resistance to flow is modeled with Equation 5.

$$
\tau=\mu p+\tau_{0}^{\prime}
$$

where $\tau_{0}^{\prime}$ is a persistent or residual cohesive strength.

Obviously, this model is not a complete treatment of all complex phenomena which may occur in the fracture and flow of soils and rocks. Coupling of volumetric strain (bulking) with shear strain through the processes of fracture, and subsequent flow, for example, is not attempted. Smooth and continuous volumetric strain hardening which may be observed in some materials test data would have to be modeled with these forms 
by two linear segments. Discrete separation of response zones which occurs because of the intersection of two linear lines in this approximate $p$ - $n$ model would not necessarily be sharply discernible in penetration tests. Frictional resistance to flow will vary with the degree of fracture and compaction but for simplicity, this factor is assumed to be constant in this model. Finely layered geologic detail and orthotropic behavior have also been cmitted for the advantages of simplicity. Strain rate effects may be important in high speed penetrations but again for simplicity, these effects are not included.

First order estimates for use on simply defined targets with attendant coverage of various factors are afforded with this model. The model is intended for use in predicting the response of projectiles penetrating into generally defined but poorly measured targets.

When the shear resistance to flow, Equation 5 is substituted, Equations 2 and 3 may be updated to:

$$
\begin{aligned}
\sigma_{r} & =\left(\frac{3+2 \mu}{3}\right) p+\frac{2 \tau_{0}^{\prime}}{3} \\
\tau & =\left(\frac{3 \mu}{3+2 \mu}\right) \sigma_{r}+\frac{3 \tau_{0}^{\prime}}{3+2 \mu} \\
\text { or } \quad \tau & =\frac{a}{2}\left(\mu \sigma_{r}+\tau_{0}^{\prime}\right)
\end{aligned}
$$

where $a=\frac{6}{3+2 \mu}$ 
Assumed Particie Velocity Distribution

Within wake of the crush Front

A simple expression for the particle velocity as a function of radial position is discussed in this section. With the use of this expression, the equation of motion may be directly integrated to achieve an economical, closed-form, solution to an otherwise complicated nonlinear equation that would require some kind of numerical solution. The simplification concerns the relative magnitude of the volumetric strain rate to the shear strain rate in the wake zones of the waning cavities. volumetric strain rate for spherical expansion ${ }^{[6]}$ is given by

$$
\dot{n}=-\left(\frac{\partial v}{\partial r}+\frac{2 v}{r}\right)
$$

and shear strain rate $e^{[6]}$ by

$$
\dot{\varepsilon}=-\left(\frac{\partial v}{\partial r}-\frac{v}{r}\right)
$$

where $(v)$ is the radially directed velocity of a material element currently at the radius $(r)$. The sign convention adopted is such that positive values of $p$ and $\tau$ will produce positive values of $\eta$ and $\varepsilon$.

The shear strain and shear strain rate are essentially unrestrained by the material model following fracture. That is, once the material's cohesive strength is broken the 
material is insensitive to further shear strain (a perfectly plastic condition).

The shear strain may be (and is) arbitrary and large and there is no motivation provided by the shear model for the material to recover the plastic shear distortion once the stresses are relaxed. The shear strain is therefore free to be as large as necessary to satisfy other governing equations.

There are three reasons why the volumetric strain rate is small in the wake zones of the smoothly waning cavity expansion problem:

1. The volumetric strain (unlike the shear strain) is upper bounded and this upper bound is approximately given by $n_{\ell}$. only under strong pressure will n greatly exceed $n_{\ell}$. In the absence of secondary shock loading or disturbances at the boundaries (inner cavity or at the advancing shock front) the volumetric strain for a given material element in the wake zones will be smoothly and monotonically declining (or slowly increasing) with time from (or toward) a relatively small peak.

2. The material in the compacted zone is relaxing in some cases and the unloading $p$ - $n$ relationship governs. As discussed in the material model section of this report, this slope is assumed to be much greater than $K$. Smooth relaxation along nearly vertical lines would produce negligibly small values of $\dot{n}$ and in the limiting case of vertical lines, this relaxation would provide the generalized locking model results used by Yarrington, [4] e.g., $\dot{n} \equiv 0.0$. 
3. The third factor governing the magnitude of the volumetric strain rate is the strength of $\ddot{q}$ (acceleration of the inner cavity radius). When $\ddot{q}$ is zero, an essentially constant velocity, one dimensional, compaction loading of the material results at the leading front and the pressure on the crushed material varies relatively slowly with time. This is more easily seen in infinitely long, rectilinear models where the material behind the leading front remains at constant velocity and the strength of the pressure at the front remains constant. In moderate velocity, deep penetrations with slender convex or conical-nosed projectiles, $\ddot{q}$ for the spherical cavity is a relatively small negative value after the step increase in velocity at the nose tip.

Any one of these reasons could be used to justify the approximation that volumetric strain rate in the crushed material is negligibly small relative to the shear strain rate. In the slender, convex-nosed penetrator problem, all three reasons support this approximation:

$$
|\dot{n}|<|\dot{\varepsilon}|
$$

For an order of magnitude examination, let the volumetric strain rate in the wake zones be represented by a spatially constant (uniformly expanding) variable in time.

$$
\dot{n}(r, t) \cong \dot{\bar{n}}(t)
$$


With the use of Equations 10 and 13, the following partial differential equation may be formed:

$$
\frac{\partial v}{\partial r}+\frac{2 v}{r}=-\dot{\bar{n}}(t)
$$

which may be integrated to yield:

$$
v=\frac{F(t)}{r^{2}}-\frac{\dot{n} r}{3}
$$

where $F(t)$ is a function of time only and the notations $F(t)$ and $F$ are interchangeable once the functional dependence is introduced. This expression for $v$ may be substituted into Equation 11 to provide

$$
\dot{\varepsilon}=\frac{3 F}{r^{3}}
$$

Substitution of.Equations 13 and 15 into Expression 12 provides

$$
|\dot{\bar{n}}| \ll\left|\frac{3 F}{r^{3}}\right|
$$

or when the signs of these functions are studied

$$
|\dot{\bar{n}}|=-\dot{\bar{n}} ; \text { volumetric strain relaxation }
$$




$$
\left|\frac{3 F}{r^{3}}\right|=\frac{3 F}{r^{3}} \quad \text {; for positive velocity field, }
$$

this expression becomes:

$$
-\dot{\bar{n}}<\frac{3 F}{r^{3}}
$$

and when this expression is multiplied through by $\frac{r}{3}$,

$$
-\frac{\dot{\bar{n}} r}{3}<\frac{F}{r^{2}}
$$

This result then illustrates the simplification of Equation 14 to

$$
v \cong \frac{F}{r^{2}}
$$

$F(t)$ is determined by setting $v=\dot{q}$ at $r=q$;

$$
F(t)=q^{2} \dot{q}
$$

Simply stated, this particle velocity distribution is the same as that which would result from the imposition of incompressibility or ideally locking material assumptions. It should be clearly understood, however, that neither of these material-based assumptions is sufficient by itself to guarantee the validity of Equation 16 for real materials. One should also recall that these results, Equations 16 and 17 , are valid only for the particular problem of smoothly waning cavity expansion and the appropriateness of these forms for more generally defined problems is not implied. 


\section{Crush Front Dynamics}

As discussed in the Introduction, the crush front conditions may be described by one of three conditions for the material model and the particle velocity at the advancing front. For convenience, these are termed: low speed, intermediate speed, and high speed crush front conditions.

\section{Low Speed Conditions}

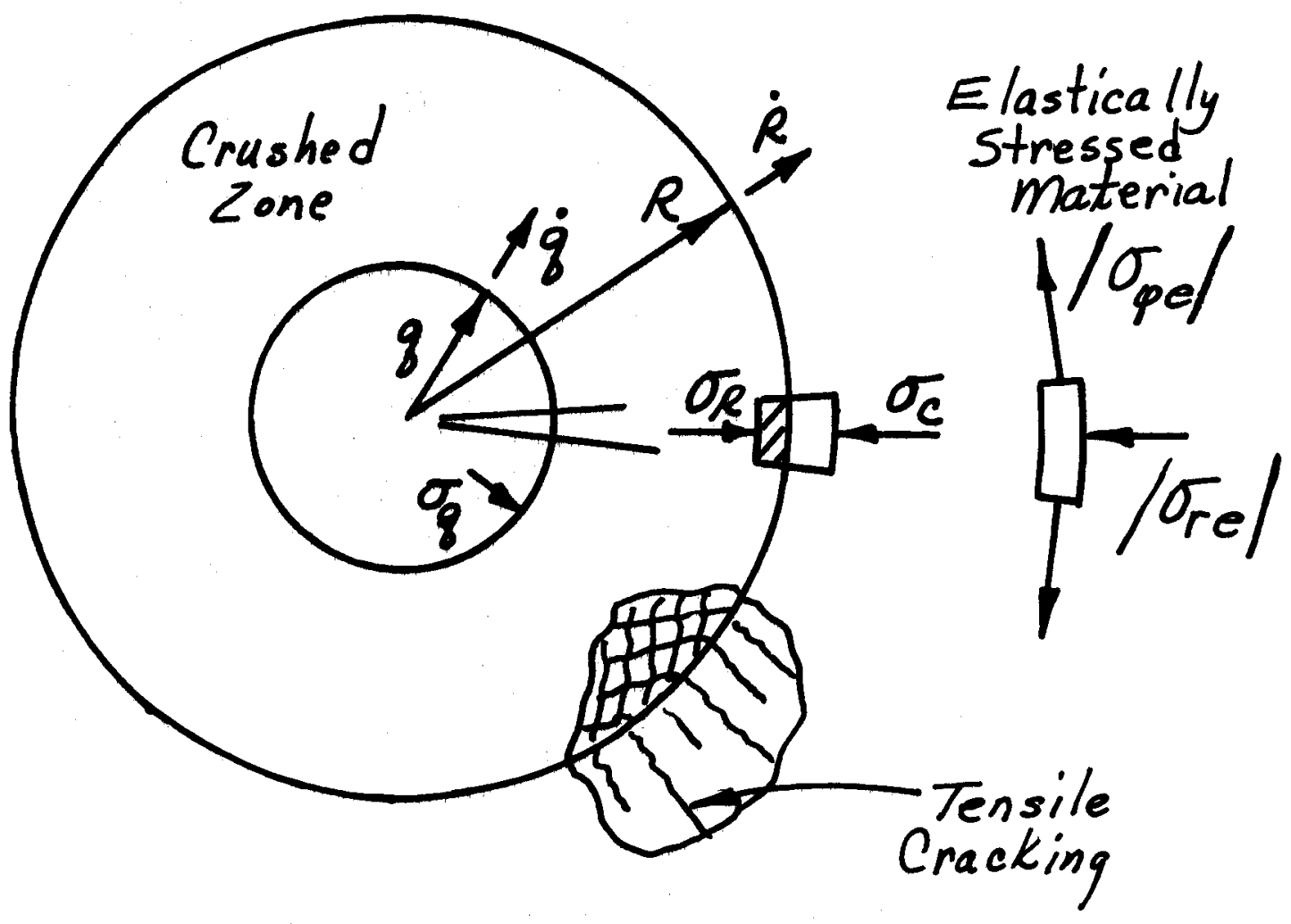

Figure 10

Approximate Crush Front Conditions for Low Speed Expansion 
Radius, $R(t)$, is assumed to separate the crushed rock zone from the elastically stressed zone in Figure 10. As the radius $R$ advances (forced by the inner cavity expansion, $\dot{q}$ ) the elastic stress field, consisting of radial compression ( $\left.\sigma_{r e}\right)$ and tangential tension $\left(\sigma_{\phi e}\right)$ stress,components, would exist in a quasi-static mode. Since the material is weak in tension, radial cracking should be expected at the inner boundary of the "elastically"stressed zone. The radial stress necessary to further crush-collapse the material under continuous growth of the radius $R$ is therefore assumed to be $\sigma_{C}$; where $\sigma_{C}$ is the uniaxial compression strength for the material or $\sigma_{C} \cong \tau_{0}$ from the material model.

The radius $R$ is determined from the conservation of mass equation. If from an expansion time, $t_{0}$, where $R=R_{0}$ and $q=q_{0}$, the crushed region grows by the addition of material compacted to a constant volumetric strain, $\eta_{R}$, then at some later time, $t$ (where $R=R, q=q$ ) this volumetric strain is given by

$$
n_{R}=\frac{R^{* 3}-R_{0}^{3}}{R^{3}-\frac{R_{0}^{3}}{5}}
$$

where $R^{\star}$ locates the current position of material which was located at radius $R_{0}$ at time $t_{0}$. This radius, $R^{*}$, is determined from the inner cavity conditions by 


$$
\stackrel{\star}{R}^{3}=R_{0}^{3}+q^{3}-q_{0}^{3}
$$

which results from the spherical expansion of material at constant density within the inner zone. Substitution of Equation 19 into 18 yields

$$
R^{3}=R_{0}^{3}+\frac{1}{n_{R}}\left(q^{3}-q_{0}^{3}\right)
$$

From the material model, Equation 6 and where

$$
\begin{aligned}
& { }_{\mathrm{R}}=\tau_{0} \\
& \mathrm{p}_{\mathrm{R}}=\frac{3}{3+2 \mu}\left[\tau_{0}-\frac{2 \tau_{0}^{\prime}}{3}\right]
\end{aligned}
$$

and

$$
\begin{aligned}
& n_{R}=p_{R} / K \\
& n_{R}=\frac{3 \tau_{0}-2 \tau_{0}^{\prime}}{K(\overline{3+2 \mu})}
\end{aligned}
$$

which is a constant function of material properties. Returning to Equation 20 and substituting the expression for $\eta_{R}$ from Equation 24 , the radius $(R)$ is then given by

$$
R=\left[R_{0}^{3}+\frac{K(3+2 \mu)\left(q^{3}-q_{0}^{3}\right)}{3 \tau_{0}-2 \tau_{0}^{j}}\right]^{1 / 3}
$$


Obviously this relationship is invalid for a material where $\tau_{0}$ and $\tau_{0}^{\prime}$ are zero (i.e., no shear strength in the absence of a confining pressure) and a response zone of this description (Zone I of Figures 2, 3 and 4 ) would not theoretically exist.

\section{Intermediate Speed Zone}

This zone, $\phi>\phi_{1}$ in Figure 4 and $\phi_{2}>\phi>\phi_{1}$ in Figure 5 , is that described in the Introduction where the expansion rate is fast enough to fracture the rock at the advancing wave front but where the pressure jump behind this front is less than $P_{\ell}$. Elastic stress and particle velocity outside the crush front are assumed to be negligible in comparison to the particle velocity and stress inside this front.

Jump conditions for conservation of mass and impulse momentum balance provide:

$$
\begin{aligned}
& \dot{R}=v_{R} / n_{R} \\
& \sigma_{R}=\rho_{0} \dot{R} v_{R}
\end{aligned}
$$

where the subscript $R$ refers to conditions inside the advancing front.

Substitution for $v_{R}$ from Equation 26 into Equation 27 yields

$$
\dot{R}^{2}=\sigma_{R} / \rho_{0} n_{R} \text {. }
$$




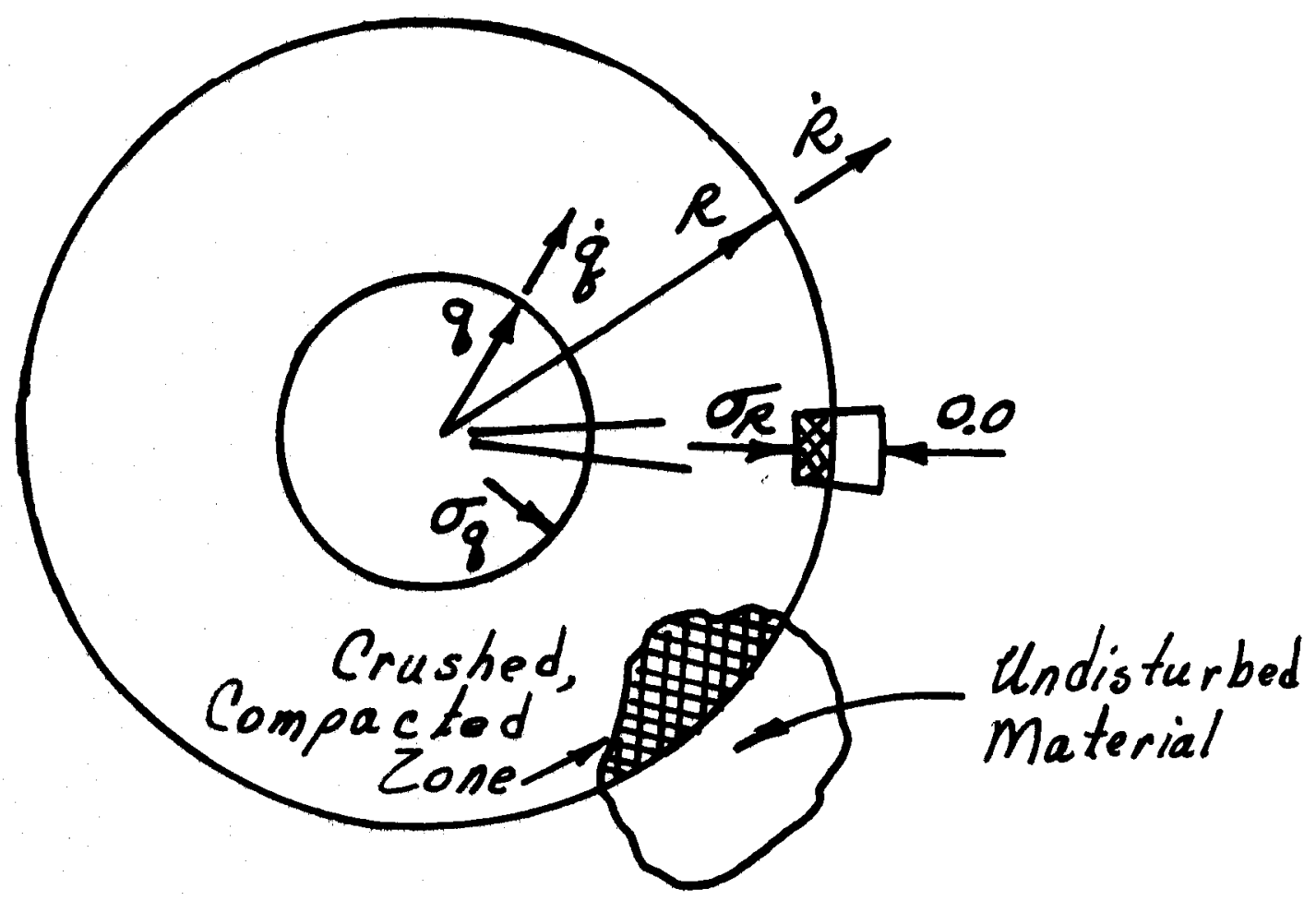

Figure 11

Approximate Crush Front Conditions for Intermediate Speed Expansion 
From the material model,

$$
{ }^{n} R=p_{R} / K
$$

and from Equation 6 ,

$$
p_{R} \equiv \frac{3 \sigma R}{3+2 \mu}
$$

when $\tau_{0}^{\prime}$ is neglected.

When these are substituted into Equation 28 , the shock front speed is determined to be

$$
\dot{R}=c
$$

where

$$
c^{2}=\frac{K(3+2 \mu)}{3 \rho_{0}}
$$

Since $c$ is a constant, the radius $(R)$ is then given by

$$
R=R_{2}+c\left(t-t_{2}\right)
$$

where $R_{2}$ is the value of $R$ at the time $\left(t_{2}\right)$ when this intermediate velocity of crushing begins. Back substitution using Equations 16, 17, 27 and 31 yields

$$
\sigma_{R}=\frac{c_{0} q^{2} \dot{q}}{R^{2}}
$$

The conditions necessary for transition from zone II response at the crush front to zone I crush front conditions occur when the frontal stress decays to the elastic limit. In equation form 


$$
\left(\sigma_{R}\right)_{I I}=\left(\sigma_{R}\right)_{I}
$$

at

$$
R=R_{1}
$$

where $R_{l}$ is that value of $R$ on Figure 4 and 5 which occurs at the time $t_{1}$.

Substitution of derived expressions for each of these stress values results ill

$$
\frac{c_{0} q^{2} \dot{q}}{R_{1}^{2}}=\tau_{0}
$$

or

$$
R_{1}=\sqrt{\frac{c_{o_{0}} q^{2} \dot{q}}{\tau_{0}}}
$$

\section{High Speed Zone}

This zone, $\phi \geq \phi_{2}$ in Figure 5 , is similar to the intermediate expansion rate case except that the shock induced pressure exceeds $p_{\ell}$, and with a corresponding volumetric strain which is very nearly $n_{\ell}$. Conservation of mass at the shock front, Equation 26 , provides

$$
\dot{R}=v_{R} / n_{l}
$$

and with the use of $v_{R}=q^{2} \dot{q} / R^{2}$

$$
R^{2} \dot{R}=\frac{1}{n_{l}} q^{2} \dot{q}
$$

or $\quad R^{2} d R=\frac{1}{n_{l}} q^{2} \cdot d q$ 
which when integrated yields

$$
R^{3}=q^{3} / n_{\ell}
$$

since, if this zone exists, $R$ must approach zero as q goes to zero; i.e., the constant of integration must vanish in this case. The radius ( $R$ ) is then

$$
R=\alpha q \text {, where } \alpha=1 / n_{\ell}^{1 / 3}
$$

and

$$
\dot{R}=\alpha \dot{q}
$$

The radial stress at radius ( $R$ ) is determined from impulse momentum relationship as before (see Equations $16,27,42,43$ )

$$
\sigma_{R}=\rho_{0}(\alpha \dot{q})\left(\frac{q^{2} \dot{q}}{\alpha^{2} q^{2}}\right)
$$

or

$$
\sigma_{R}=\frac{\rho_{0} \dot{q}^{2}}{a}
$$

The conditions necessary for transition from Zone III response at the crush front to zone II crush front (along $R$ ) occur when the shock loading decays to the level found for zone II; i.e.,

$$
\left(\sigma_{R}\right)_{\text {I I I }}=\left(\sigma_{R}\right)_{\text {II }} \text {, at } R=R_{2}
$$

Substitution of these derived expressions (Equations 32, 44) into Equation 45 yields 


$$
\dot{\mathrm{q}}_{\mathrm{cr}}=\frac{\mathrm{c}}{\alpha}
$$

The existence of Zone III response is therefore governed by inner cavity velocities which exceed the material constant $(c / \alpha)$; i.e., for that range of velocities (normal to the nose tip) where

$$
\dot{q} \geq \frac{c}{\alpha}
$$

Note that for a "perfectly distended-locking material model," $K$ is zero, $c$ is zero and $\dot{q}$ always satisfies the required condition for existence of zone III response. 
For spherical expansion, the differential equation of motion is given by

$$
\frac{\partial \sigma r}{\partial r}+\frac{2\left(\sigma_{r}-\sigma_{\phi}\right)}{r}=-\rho \frac{d v}{d t}
$$

When Equations 2 and 8 are substituted, the Equation of motion becomes

$$
\frac{\partial \sigma r}{\partial r}+\frac{\mu \alpha \sigma r}{r}=\frac{-a \tau_{0}^{\prime}}{r}-\rho \frac{d v}{d t}
$$

The particle acceleration, $\frac{d v}{d t}$, is determined from the particle velocity with

$$
\frac{d v}{d t}=\frac{\partial v}{\partial t}+v \frac{\partial v}{\partial r}
$$

When the approximate expression for the particle velocity, $v=F / r^{2}$, is substituted.

$$
\frac{d v}{d t}=\frac{\dot{F}}{r^{2}}-\frac{2 F^{2}}{r^{5}}
$$

where

$$
\dot{F}=q^{2} \ddot{q}+2 q \dot{q}^{2}
$$

is found by equating particle acceleration to cavity acceleration at $r=q$. When the density in each zone (i) is approximated with an average density, $\bar{p}_{j}$, the equation of motion is placed in an integrable form

$$
\frac{\partial \sigma_{r}}{\partial r}+\mu a \frac{\sigma_{r}}{r}=\frac{-\mathrm{a} \tau^{\prime}}{{ }^{\prime}}-\bar{\rho}_{i}\left(\frac{\dot{\mathrm{F}}}{r^{2}}-\frac{2 F^{2}}{r^{5}}\right)
$$


The solution for $\sigma_{r}$ is of the form

$$
\sigma_{r}=A(t) r^{-b}+B_{i}(r, t)
$$

where

$$
b=\mu a
$$

$A(t)$ is an unknown time dependent function and $B_{j}(r, t)$ takes on various forms which depend on the material property $\mu$.

Case 1. $\quad \mu \neq 0, b \neq(1$ or 4$)$

$$
B_{i}(r, t)=\frac{-\tau_{0}^{\prime}}{\mu}-\bar{o}_{i}\left[\frac{\dot{F}}{(b-T) r}+\frac{2 F^{2}}{(4-b) r^{4}}\right] .
$$

Case 2. $\quad \mu=0, b=0, a=2$

$$
B_{i}(r, t)=-2 \tau_{0}^{\prime} \quad \ln r-\bar{p}_{i}\left[\frac{-\dot{F}}{r}+\frac{F^{2}}{2 r^{4}}\right]
$$

Case 3. $\quad b=1, \mu=3 / 4, a=4 / 3$

$$
B_{i}(r, t)=\frac{-4 \tau_{0}^{\prime}}{3}-\bar{r}_{i}\left[\frac{\dot{F} \ln r}{r}+\frac{2 F^{2}}{3 r^{4}}\right]
$$

Note that the singular condition which would occur for b equal to four is never encountered when $\mu$ is limited to only zero or positive values.

Composite solutions for spherically expanding cavities which correspond to the various penetrator velocity conditions; Figures 3,4 and 5 are now developed in increasing order of speed. 
Slow Speed Penetration Cavity Solution, Figure (3)

The function of time, $A(t)$ is named $A_{l}$ for this case and it is determined by substituting

$$
\sigma_{R}=\tau_{0}\left(\tau_{0}>0 \text { is required }\right) \text {, Equation } 21 \text { into Equation }
$$

54, i.e.,

$$
\tau_{0}=A_{1} R^{-b}+B_{1}(R, t)
$$

where

$$
R=\left[\frac{K(3+2 \mu)}{3 \tau_{0}-2 \tau_{0}^{\prime}}\right]^{1 / 3} q
$$

is found from Equation 25 where $R_{0}=q_{0}=0$. The expression for $A_{l}$ is then given by

$$
A_{1}=R^{b}\left[\tau_{0}-B_{1}(R, t)\right]
$$

The radial stress at the inner cavity, $\sigma_{q}$, is then given by Equation 54

$$
\sigma_{q}=\left(\frac{R}{q}\right)^{b}\left[\tau_{0}-B_{1}(R, t)\right]+B_{1}(q, t)
$$

where $B_{1}(r, t)$ has one of the material dependent functional forms, Equation 56, 57 or 58 and where the average density is given by

$$
\bar{\rho}_{1}=\frac{\rho_{0} R^{3}}{R^{3}-q^{3}}
$$

Intermediate Speed Range, Figure 4

With reference to Figure 4 two separate regions, $\phi \geq \phi_{1}$ and $\phi<\phi_{1}$, must be studied. 


$$
[\phi \geq \phi 1]
$$

In this crushed zone, zone II only, radial stress is given by Equation 54

$$
\sigma_{r}=A_{2}(t) r^{-b}+B_{2}(r, t)
$$

The function of time, $A_{2}(t)$, is determined by the stress condition given by Equation 34

$$
\sigma_{R}=\frac{c \rho_{0} q^{2} \dot{q}}{R^{2}}
$$

at $R=c t$

and where $c$ is determined by Equation 32 and where $t$ is the time of expansion. The function, $A_{2}(t)$ is then determined from Equations 34 and 63 to be

$$
A_{2}=R^{b}\left[\frac{C_{o_{0} q^{2} \dot{q}}}{R^{2}}-B_{2}(R, t)\right]
$$

The radial stress at the inner cavity, $\sigma_{q}$, is then given by Equation 54

$$
\sigma_{q}=A_{2} q^{-b}+B_{2}(q, t)
$$

or when Equation 64 is substituted for $A_{2}$.

$$
\sigma_{q}=\left(\frac{R}{q}\right)^{b}\left[\frac{c_{o} q^{2} \dot{q}}{R^{2}}-B_{2}(R, t)\right]+B_{2}(q, t)
$$


where $B_{2}(r, t)$ has one of the material dependent functional forms, Equations 56,57 , or 58 and where the average density is given by

$$
\begin{aligned}
& \bar{\rho}_{2}=\frac{\rho_{0} R^{3}}{R^{3}-q^{3}} \\
& {\left[\phi \leq \phi_{1}\right]} \\
& \text { Radial stress in Zone I is provided by Equation } 54 \\
& \sigma_{r}=A_{1} r^{-b}+B_{1}(r, t)
\end{aligned}
$$

where $A_{l}(t)$ is determined with Equations 21 and 54

$$
A_{1}=R^{D}\left[\tau_{0}-B_{1}(R, t)\right]
$$

and

$$
R=\left[R_{1}^{3}+\frac{k(3+2 u)\left(q^{3}-q_{1}^{3}\right)}{3 \tau_{0}-2 \tau_{0}^{1}}\right]^{1 / 3}
$$

is found with Equation 25. $B_{1}(r, t)$ has one of the material dependent functional forms, Equations 56,57 , or 58 and where the average density is given by

$$
\bar{o}_{1}=\frac{\rho_{0}\left(R^{3}-R_{1}^{3}\right)}{R^{3}-S^{3}}
$$

The radial stress at the surface $(S)$ is then expressed with Equation 54 as 


$$
\sigma_{s}=A_{1} S^{-b}+B_{1}(S, t)
$$

Radial stress within Zone II is expressed by Equation 54 as

$$
\sigma_{r}=A_{2} r^{-b}+B_{2}(r, t)
$$

and at the surface (S) with

$$
\sigma_{S}=A_{2} S^{-b}+B_{2}(S, t)
$$

Solving for $\sigma_{s}$ from Equations 70 and 71 yields

$$
A_{2}=A_{1}+S^{b} \cdot\left[B_{1}(S, t)-B_{2}(S, t)\right]
$$

The radial stress at surface $(q)$ is then determined with Equations 54 and 72 to be

$$
\sigma_{q}=A_{i} q^{-b}+\left(\frac{S}{q}\right)\left[B_{1}(s, t)-B_{2}(s, t)\right]+B_{2}(q, t)
$$

Substitution for $A_{1}$ from Equation 67 yields

$$
\begin{aligned}
\sigma_{q}= & \left(\frac{R}{q}\right)^{b}\left[\tau_{0}-B_{1}(R, t)\right]+\left(\frac{S}{q}\right)^{b}\left[B_{1}(S, t)-B_{2}(S, t)\right] \\
& +B_{2}(q, t)
\end{aligned}
$$

In the above, $B_{2}(r, t)$ is the same material dependent functional form as $B_{j}(r, t)$ except it has an average density given by

$$
\bar{p}_{2}=\frac{\rho_{0} R_{1}^{3}}{R_{1}^{3}-q_{1}^{3}}
$$


The radius(S) is defined by expanding a constant density, constant amount of material, i.e., the amount of material in Zone II of Figure 4 is unchanging for $\phi<\phi_{1}$.

$$
S=\left[R_{1}^{3}+\left(q^{3}-q_{1}^{3}\right)\right] 1 / 3
$$

Intersection of surfaces $S$ and $R$ is determined by

$$
S=R=R_{1}
$$

where $R_{1}$ is found by simultaneously solving the two functions:

$$
R=\sqrt{\frac{c_{0} q^{2} \dot{q}}{\tau_{0}}} \text {, from Equation } 37 \text { (where } q \text { and } \dot{q} \text { are }
$$

prescribed functions of time) and

$$
R=c t \text {. }
$$

Graphically, this is the intersection of two time dependent curves as illustrated as follows:

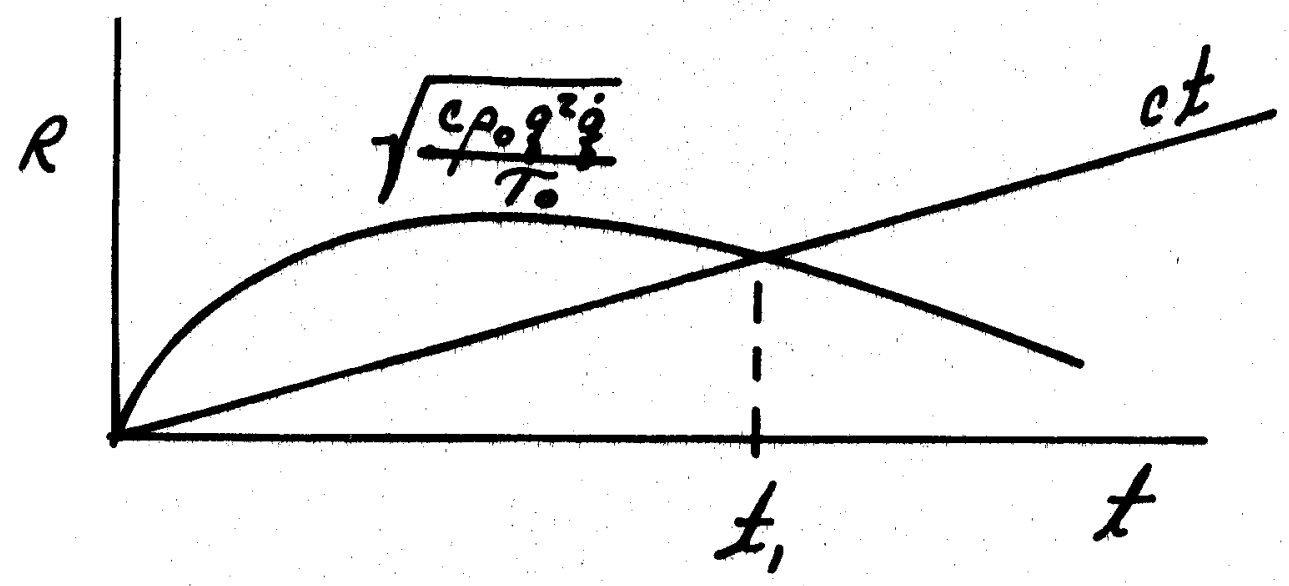

and

$$
R_{1}=c t_{1}
$$


High Speed Penetration, Figure 5

With reference to Figure 5 three separate regions $\left(\phi \geq \phi_{2}\right.$, $\phi_{2}>\phi_{2} \phi_{1}$, and $\phi<\phi_{1}$ ) must be studied. The methodology necessary to develop composite solutions for these three regions for this high speed case is the same as that used in the intermediate speed case. Results of this development are listed below:

$$
\begin{aligned}
& {\left[\phi \geq \phi_{2}\right]} \\
& \quad \sigma_{q}=\alpha^{b}\left[\frac{\rho_{0} \dot{q}^{2}}{\alpha}-B_{3}(R, t)\right]+B_{3}(q, t)
\end{aligned}
$$

where

$$
\begin{aligned}
& R=\alpha q \\
& \bar{\rho}_{3}=\frac{p_{0}}{\Gamma_{l}}
\end{aligned}
$$

and $B_{3}(r, t)$ is one of the material dependent functional forms, Equations 56,57 , or 58 .

$$
\begin{aligned}
& {\left[\phi_{2}>\phi \geq \phi_{1}\right]} \\
& \sigma_{q}=\left(\frac{R}{q}\right)^{b}\left[\frac{C_{0} q^{2} \dot{q}}{R^{2}}-B_{2}(R, t)\right]+\left(\frac{Q}{q}\right)^{b}\left[B_{2}(Q, t)-B_{3}(Q, t)\right] \\
& +B_{3}(q, t)
\end{aligned}
$$


When the transitional radius $\left(R_{2}\right)$ is determined from

$$
R_{2}=\alpha q_{2}
$$

where $q_{2}$ is that value of $q(t)$ which coincides with the velocity condition

$$
\dot{q}=\frac{c}{\alpha}
$$

the terms in Equation 80 are given by

$$
\begin{aligned}
& R=R_{2}+c\left(t-t_{2}\right) \\
& Q=\left[R_{2}^{3}+\left(q^{3}-q_{2}^{3}\right)\right]^{1 / 3} \\
& \bar{\rho}_{2}=\frac{\rho_{0}\left(R^{3}-R_{2}^{3}\right)}{R^{3}-Q^{3}} \\
& \vec{\rho}_{3}=\frac{\rho_{0}}{1-n_{l}}
\end{aligned}
$$

$B_{2}(r, t)$ and $B_{3}(r, t)$ are the same material dependent functional forms from Equations 56,57 or 58 except different average densities are asked for by the subscript notation.

$$
\left[\begin{array}{lll}
\phi & < & \phi
\end{array}\right]
$$

The radius $(R)$ is determined by solving the following equations. Time at transition $\left(t_{1}\right)$ is first determined by solving 


$$
R_{2}+c\left(t_{1}-t_{2}\right)=\sqrt{\frac{c \rho_{0} q_{1}^{2} \dot{q}_{1}}{\tau_{0}}}
$$

where $R_{2}$ is that value determined by Equation 81 and $q_{1}$ and $\dot{q}_{1}$ are the values of the prescribed function of time $(q(t)$ and $\dot{q}(t))$ which satisfy this equation. The outer radius $\left(R_{p}\right)$ at transition time $\left(t_{1}\right)$ is then

$$
R_{1}=R_{2}+c\left(t_{1}-t_{2}\right)
$$

and at a later instant in time the outside radius is given by appropriate substitution of variables into Equation 25

$$
R=\left[R_{1}^{3}+\frac{k(3+2 \mu)\left(q^{3}-q_{1}^{3}\right)}{3 \tau_{0}-\frac{2 \tau_{0}^{\prime}}{1 / 3}}\right]^{1}
$$

The radius ( $S$ ) is calculated from Equation 75

$$
S=\left[R_{1}^{3}+\left(q^{3}-q_{1}^{3}\right)\right]^{1 / 3}
$$

The radius (Q) is calculated from

$$
Q=\left[R_{2}^{3}+\left(q^{3}-q_{2}^{3}\right)\right]^{1 / 3}
$$

and at transition time

$$
Q_{1}=\left[R_{2}^{3}+\left(q_{1}^{3}-q_{2}^{3}\right)\right]^{1 / 3}
$$

Average densities for the material in the separate zones 
for $\phi<\phi_{1}$ are

$$
\bar{\rho}_{1}=\rho_{0}\left(\frac{R^{3}-R_{1}^{3}}{R^{3}-S^{3}}\right), \bar{\rho}_{2}=\rho_{0}\left(\frac{R_{1}^{3}-R_{2}^{3}}{R_{1}^{3}-Q_{1}^{3}}\right), \bar{\rho}_{3}=\frac{\rho_{0}}{T-n_{l}}
$$

With these functions, radial stress at the inner surface (q) is given by

$$
\begin{aligned}
\sigma_{q}= & \left(\frac{R}{q}\right)^{b}\left[\tau_{0}-B_{1}(R, t)\right] \\
& +\left(\frac{S}{q}\right)^{b}\left[B_{1}(s, t)-B_{2}(s, t)\right] \\
& +\left(\frac{Q}{q}\right)^{b}\left[B_{2}(Q, t)-B_{3}(Q, t)\right]+B_{3}(q, t)
\end{aligned}
$$

where the material dependent functional forms for $B_{1}(r, t)$, $B_{2}(r, t)$ and $B_{3}(r, t)$ are the same case selected from Equations 56,57 , or 58 except for the different expressions for average densities which are $\bar{\rho}_{1}, \bar{\rho}_{2}$ and $\bar{p}_{3}$, respectively.

Equations 77,80 and 93 are general solutions for all penetration velocities since the intermediate and slow speed ranges are simply special cases.

This can be checked by deriving the intermediate speed solutions from Equation 93 by setting

$$
R_{2}=t_{2}=0
$$


and

$$
Q=q
$$

and the slow speed solution from the intermediate solution by further setting

$$
R_{1}=t_{1}=0
$$

and

$$
S=q
$$

Equation 93 is therefore the most useful form for computer programming. 
Preliminary Applications

Since several assumptions were made in the development of the solution forms of this report, results from some early applications are presented to show the degree of correlation of these methods with other solution forms and in some cases with experiment.

The most critical assumption concerns that of particle velocity distribution within the wake zone of slowly waning expansions. The appropriateness of this assumption for Zone II behavior (see Figure 4) is of particular interest. Fortunately, this assumption can be tested on water entry problems. Water is an interesting choice for several reasons: water properties are well known and water is a special case of the material model $\left(\tau_{0} \equiv \tau_{0}^{\prime} \equiv \mu \equiv 0.0\right)$ physically, water is far removed from that of a locking solid (validity of $v=F / r^{2}$ rests totally on kinematical forcing arguments) and finally, penetration into water targets has been and continues to be of strong interest. Blunt, doubly curved bodies are of interest in water impact as well and this shape offers a severe test of the unloading rate approximation (the normal component of velocity $V_{n}$ in the spherical cavity application can be greatly varied with a spherical nose and with lightweight bodies, the acceleration term $\dot{v}$ can be magnified). Case I, which follows, is for water entry/impact. 
other assumptions which remain to be tested are not so easy to verify. These primarily concern the quantification of the soil and rock material model. Cases II and III, below, are preliminary results obtained from the early version of the GNOME code. [9] Interactions of such factors as nose shape (ogive or cone together with or without separated flow) unconfined shear strength $\left(\tau_{0}\right)$ and waterial frictional resistance to flow $(\mu)$ are difficult to separate and to decisively quantify in these exercises. Here, some preliminary comparisons are illustrated to show the importance of these various factors.

\section{Case I. Water Entry}

Consider the case of a rigid sphere of radius $q$ and mass $M$ impacting water. When $T_{0}=T_{0}^{\prime}=\mu=0.0$ are substituted into equations 65 and 57 , the following equation results:

$$
\sigma_{q}=c \rho_{0} \beta^{2} \dot{q}+2 \bar{\rho}\left[\frac{3}{4}-\beta+\frac{\beta^{4}}{4}\right] \dot{q}^{2}+\bar{\rho}(1-\beta) q q
$$

When $\dot{q}=V \cos \phi$

$$
q \ddot{q}=-v^{2} \sin ^{2} \phi+q \dot{v} \cos \phi
$$

are substituted

$$
\sigma_{q}=(\bar{A}+\bar{D} \dot{V}) \cos \phi+B \cos ^{2} \phi-\bar{C} \sin ^{2} \phi
$$

\footnotetext{
* Note that angle $\phi$ and $\phi$ are specially defined for this example problem.
} 


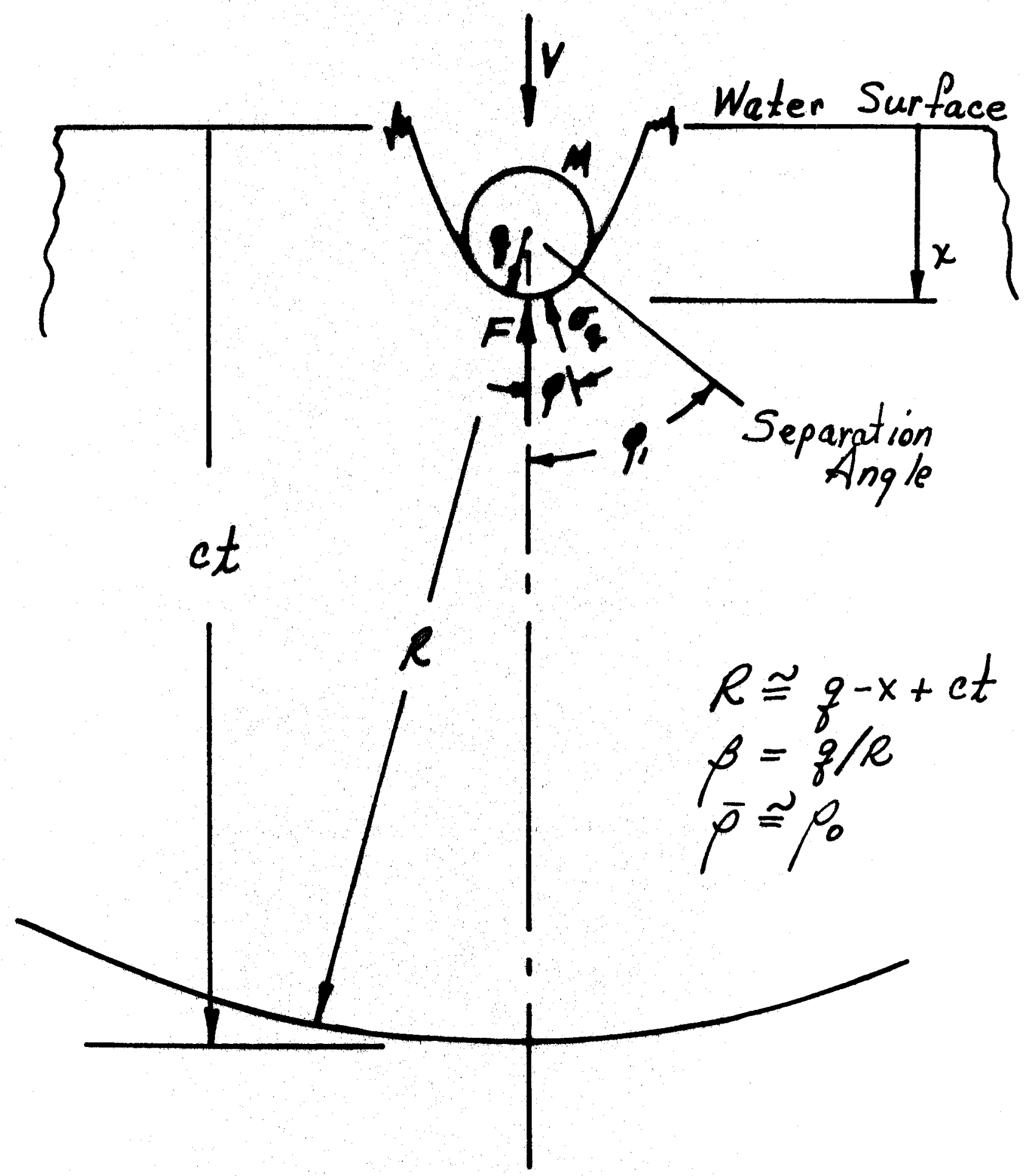

56 
where

$$
\begin{aligned}
& \bar{A}=\bar{A}(V, t)=C_{0} \rho^{2} V \\
& \bar{B}=\bar{B}(V, t)=2 \rho_{0}\left(\frac{3}{4}-\beta+\frac{\beta^{4}}{4}\right) V^{2} \\
& \bar{C}=\bar{C}(V, t)=\rho_{0}(1-\beta) V^{2} \\
& \bar{D}=\bar{D}(q, t)=\rho_{0}(1-\beta) q
\end{aligned}
$$

When this expression for $\sigma_{q}$ is integrated over the axially projected area defined by $0 \leq \phi \leq \phi_{1}$ the drag force is found to be

$$
\begin{aligned}
F= & 2 \pi q^{2}\left[\frac{1}{3}(\bar{A}+\bar{D} \dot{V})\left(1-\cos ^{3} \phi_{1}\right)\right. \\
& \left.+\frac{\bar{B}}{4}\left(1-\cos ^{4} \phi_{1}\right)-\frac{\bar{C}}{4}\left(\sin ^{4} \phi_{1}\right)\right]
\end{aligned}
$$

The angle $\phi_{1}$ is defined in "late" time as the separated flow angle, $\phi_{s}$ (where $\sigma_{q}=0.0$ at $\phi=\phi_{s}$ ) which is found from

$$
\cos \phi_{s}=-(\bar{A}+\bar{D} \dot{V})+\frac{\sqrt{(\bar{A}+\bar{D} \dot{V})^{2}+4 \bar{C}(\bar{B}+\bar{C})}}{2(\bar{B}+\bar{C})}
$$

Prior to separated flow, $\phi_{1}$ is assumed to be $\phi_{E}$

where $\quad \cos \phi_{E}=1-\frac{x}{q}$

and

$$
\phi_{1}=\phi_{E} \text { for } \phi_{E} \leq \phi_{S}
$$

57 


$$
\phi_{1}=\phi_{S} \text { for } \phi_{S} \leq \phi_{E}
$$

The equation of motion for the sphere then provides

$$
\dot{v}=\frac{-2 \pi q^{2}\left[\frac{\bar{A}}{3}\left(1-\cos ^{3} \phi_{1}\right)+\frac{\bar{B}}{4}\left(1-\cos ^{4} \phi_{1}\right)-\frac{\bar{C}}{4} \sin ^{4} \phi_{1}\right]}{M+\frac{2 \pi q^{2} \bar{D}}{3}\left(1-\cos ^{3} \phi_{1}\right)}
$$

This equation was numerically integrated ${ }^{[10]}$ over time from the initial conditions: $V=V_{0}$ and $X=0.0$ to achieve the family of curves shown in Figure 12 . These are compared with the inset family of curves which are reproduced from Reference 11. Note the curves from Reference 11 are valid on ly for the impact (entry) times $(x / q<0.25)$ since sustained cavity flow drag is not included in this prediction technique. The present method predicts both impact and a level of sustained cavity flow drag. The magnitude of this sustained part is unconfirmed at this time but the magnitudes are reasonable. The effect of body acceleration on the entry force is illustrated by the various weights (parameter $\sigma$ ) and this acceleration is more important in the sustained drag phase. Figure 13 is a comparison of peak entry acceleration for a particular body weight and two spherical radii over a range of impact velocities for the spherical cavity model and the theory of Reference 11. The expanding cavity method compares well with these references. 


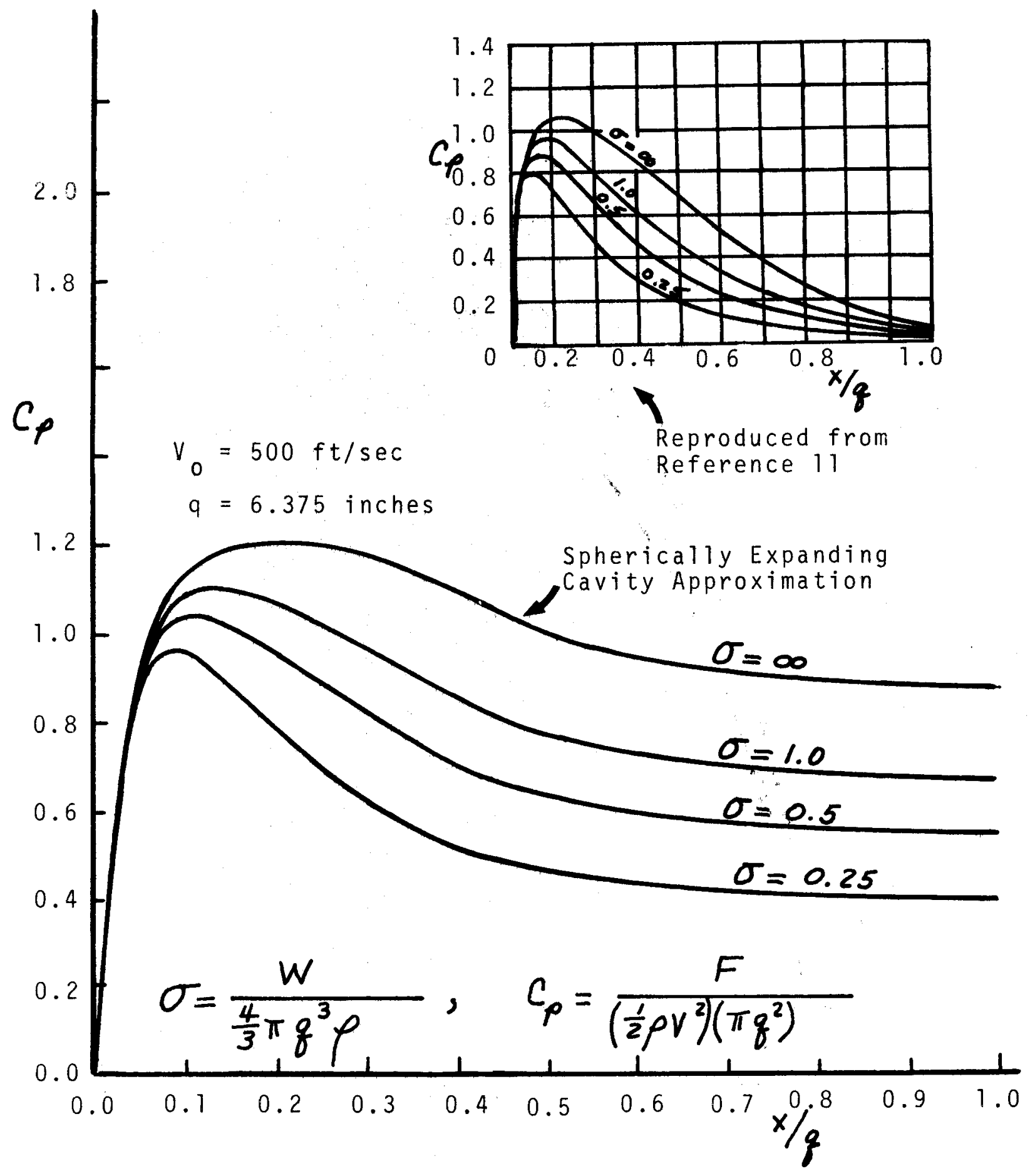

Figure 12

Water Entry Impact Force on Spherical Nose Projectile, Theoretical Comparison [10] 


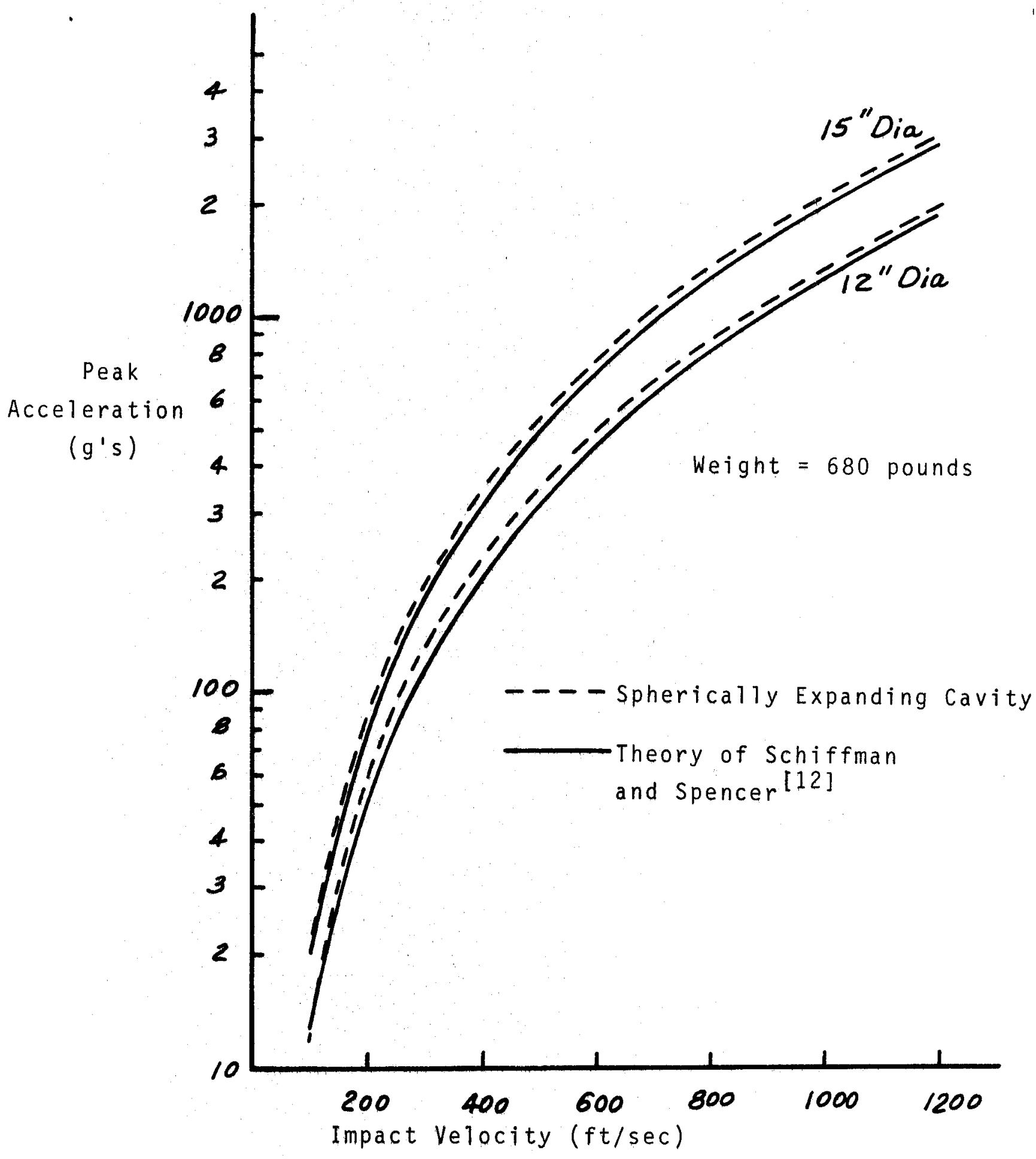

Figure 13

Comparison of Peak Acceleration on Spheres Impacting Water[10] 
Case II. Antelope Tuff/DGU 12 Test

The following material properties were reduced from measurements provided by [14]:

$$
\begin{aligned}
& \rho_{0}=1.634 \times 10^{-4} \mathrm{bb} \times \mathrm{sec}^{2} / \mathrm{in}^{4}, \mu=1.0 \\
& \tau_{0}=2,000.0 \mathrm{psi}, K=290,000.0 \mathrm{psi} .
\end{aligned}
$$

Values of $\tau_{0}^{\prime}=0.0$ and $\eta_{l}=0.25$ were assumed. Figure 14 is a plot of computed nose force on a $6 \mathrm{CRH}, 6$ inch diameter base, ogive penetrating antelope tuff as the velocity is varied. Vertical lines on this graph define the velocities which separate the cavity initiation zones depicted in Figures 3,4 , and 5. That is, Zone $i$ of Figure 14 corresponds to the condition depicted in Figure 3 ; Zone $i \mathrm{i}$ corresponds to the condition depicted in Figure 4 and Zone $\mathrm{i} i \mathrm{i}$ corresponds to the condition shown in Figure 5 .

The nose farce predicted by the methods derived in this report, for this target material, is a nonlinear function of penetratar speed. This is particularly true in the neighborhood of the DGU-12 test velocity. The axial acceleration of the penetrator measured in the DGU-12 test is plotted in Figure 15.

Preliminary results predicted by GNOME are shown for comparison. A coefficient of sliding friction of 0.1 on the 


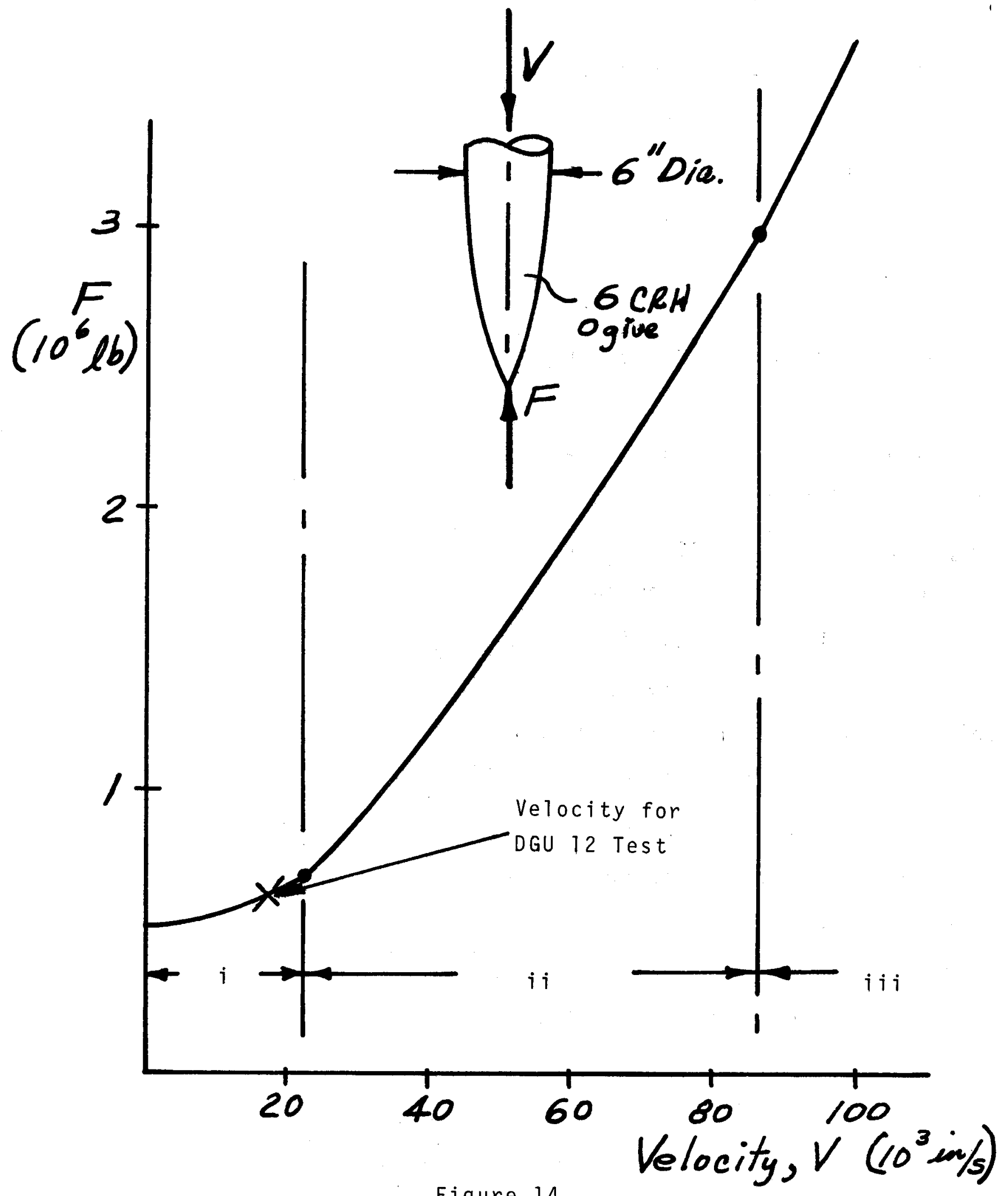

Figure 14

Ogival Nose Penetration Force into Antelope Tuff As A Function of Velocity of Penetration [9] 


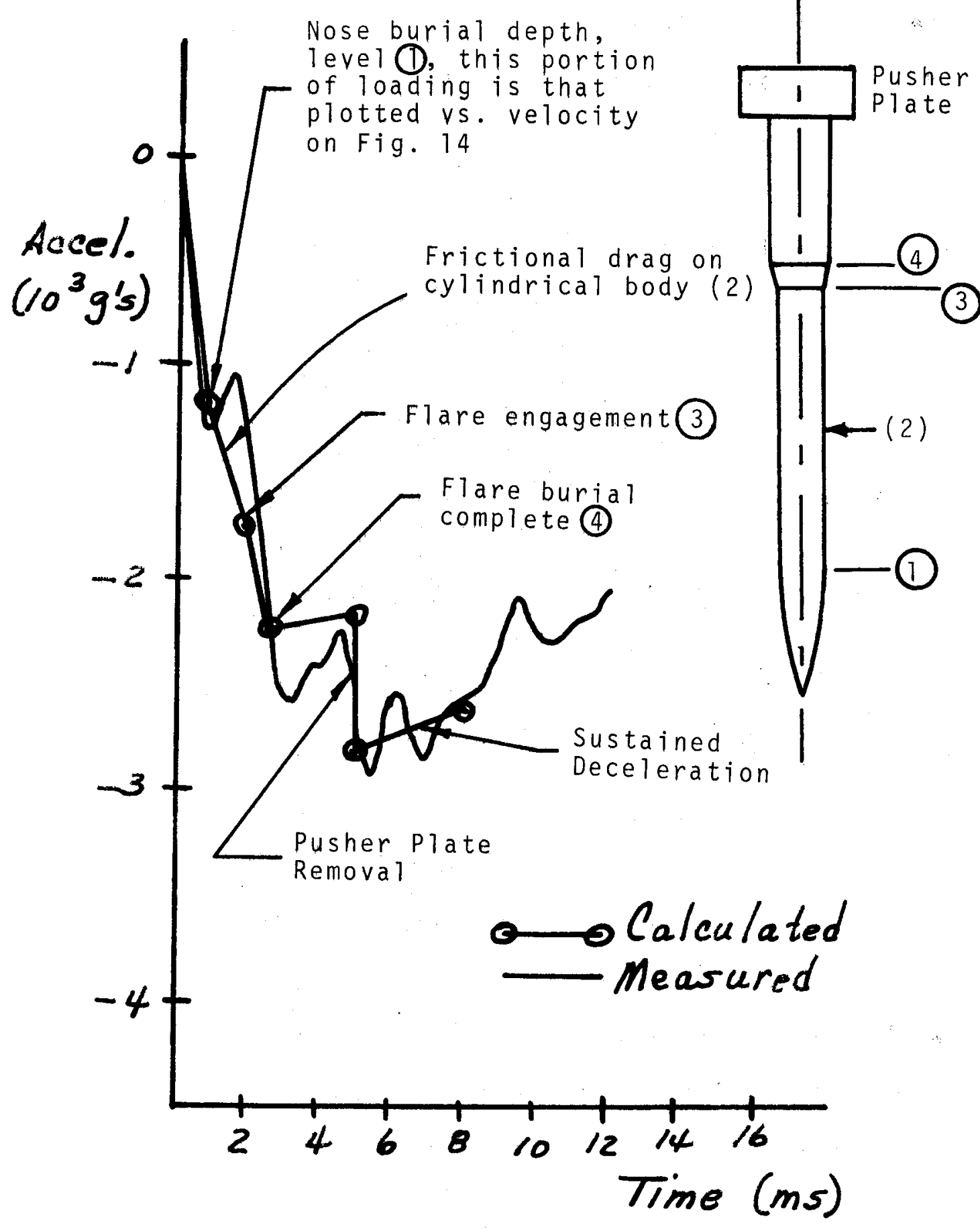

Figure 15

Acceleration-time Measurements and Predictions

For a. Field Test, Antelope Tuff, DGU-12 [9] 
cylindrical parts of the penetrator body was assumed for the complete set of calculations. In this analysis, a positive pressure was found at the tangent point; nose to cylinder junction. Separated flow did not occur and the pressure calculated at the nose transition station was assumed to act uniformly on the cylindrical body. The force at the nose burial depth is the value shown in Figure 14 .

Case II I. Mount Helen Welded Tuff

Data for Mount Helen Welded Tuff and penetrator acceleration data ${ }^{[7]}$ were studied with the preliminary coding for GNOME and the spherical cavity model described in this report. The penetrator in this test is defined by: ${ }^{[7]}$ :

Total Length $=60.0$ inches, Outer Diameter $=6.5$ inches, Ogive Nose $=9.25 \mathrm{CRH}$, Total Weight $=400.01 \mathrm{~b}$. Impact Velocity $=1,350.0 \mathrm{ft} / \mathrm{sec}$, Pusher Plate* Wejght $=$ $117.01 \mathrm{~b}$.

The accelerometer data shown with the solid lines in figure 16 are those provided by [7]. The "prediction" curve was provided with the theory of Reference 7 where it was applied to an "equivalent" conical nose having a half angle $\phi=$ $\tan ^{-1}(1 / 4)$ and where the material properties were:

ॠA pusher plate which mates with the internal diameter of the Davis gun barrel is attached to and follows the penetrator until this plate strikes the target. 


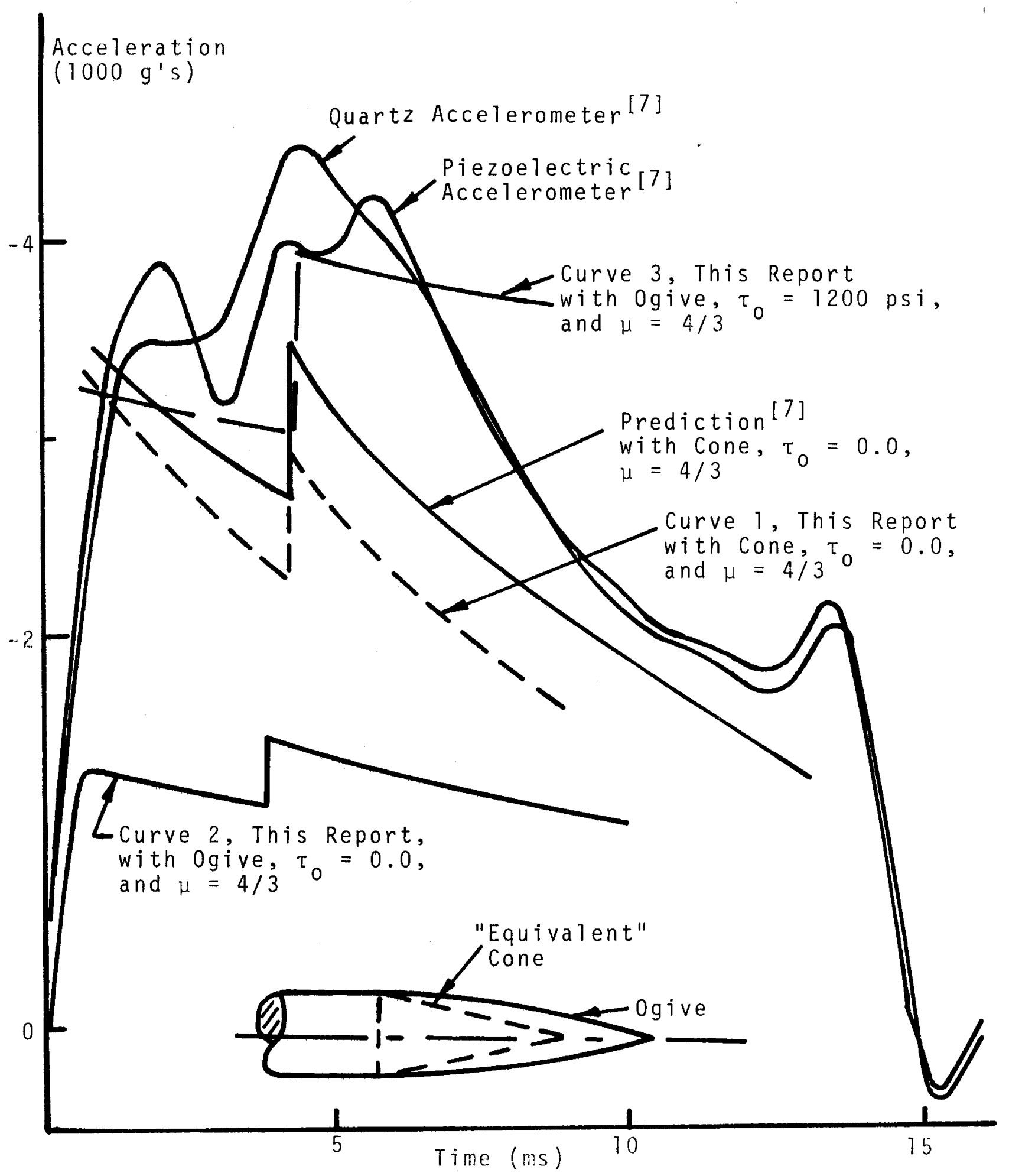

Figure 16

Acceleration-time Measurements and Predictions for a Field Test, Mount Helen Welded Tuff [9 


$$
\begin{aligned}
& \rho_{0}=1.8 \times 10^{-4} \mathrm{lb} \times \mathrm{sec}^{2} / \mathrm{in}^{4}, \mathrm{~K}=1.38 \times 10^{6} \mathrm{psi}, \\
& \mu=\frac{4}{3},{ }_{0}{ }_{0}=T_{0}^{\prime}=0.0 .
\end{aligned}
$$

For comparison purposes, these same data (including the conical nose approximation) were used in GNOME and the results are shown as curve numbered 1 on Figure 16.

An additional calculation was made where the same material properties and data were applied to the test (ogival) nose shape. Curve numbered 2 on Figure 16 shows the results.

Another calculation was made where the properties were changed to include a small value of unconfined shear strength while maintaining the same value of internal friction,

$$
\begin{aligned}
\tau_{0} & =1,200 \text { psi } \\
\mu & =\frac{4}{3}
\end{aligned}
$$

GNOME then produced curve numbered 3 for the ogival shape. The significance of the interactive effects of unconfined shear strength and the nose shape on the penetrator loading via these methods is evident. 
Conclusions

1. Computed results using the methods derived in this report indicate that nose shape, unconfined shear strength, internal material friction and slow speed solution techniques (Zone I response) are important in the prediction of penetrator forces into rock targets. Data taken from the two earth penetration tests generally support these results. Satisfactory treatment of these details is expected to be of equal importance in the prediction of lateral loading.

2. The simplifying assumptions which were made for particle velocity and density distributions introduced only small deviations from previously established results for water impact loads. The deviations appear to be improvements and the economical advantages obtained with the assumptions are noteworthy.

3. These solution forms appear useful for continued applications and testing in the new earth penetrator trajectory code GNOME and in water entry dynamics. 


\section{Nomenclature}

\section{Penetrator Variables}
D -
Diameter of cylindrical body
$L$ - Nose length
$V$ - Penetrator velocity
$v_{n}-\quad V e l o c i t y$ component normal to nose surface
$\dot{v}$ - Penetrator acceleration

Coordinates and Geometry Variables

$r, \phi, \theta$ - Polar spherical coordinates

q - Spherical cavity radius

$\dot{q}$ - Spherical cavity wall velocity

$\dot{q}_{c r}-\quad$ Value of $q$ defined by Equation 46

$\ddot{q}$ - Spherical cavity acceleration

Q - Spherical radius which divides response zone II

and Zone III

$R$ - Outermost spherical radius of crushed or compacted material

$R_{0}, R^{*}$ - Values of radius $R$ at temporary steps in the derivation

$R_{1}, R_{2}$ - Transitional values of $R$, see figure 5

$\dot{R}$ - Time rate of change of $R$

S - Spherical radius which divides Zone I and Zone II response

$\phi_{1}, \phi_{2}$ - Transitional angles, see Figure 5

$t$ - Expansion time 
$t_{1}, t_{2}$ - Transitional times which correspond with $\phi_{1}$ and $\phi_{2}$ and $R_{1}$ and $R_{2}$

a - Ratio R/q for Zone III response, Equation 42

Stress, Strain and Other Response Variables

$\sigma_{r}, \sigma_{\phi}, \sigma_{\theta}$ - Stress components in polar spherical coordinates

$\sigma_{q}-\quad$ Radial stress at cavity radius

$\sigma_{R}$ - Radial stress just inside radius $R$

$\sigma_{S}$ - Radial stress at radius $S$

$\left|{ }^{\sigma} \phi e\right|$ - Absolute value of tangential stress in elastic response zone

forel - Absolute value of radial stress in elastic response zone

$T$ - Stress difference

p - Pressure (average stress)

$p_{R}$ - Pressure (average stress) just inside radius $R$

$\eta$ - Volumetric strain, compression is positive

$\eta_{R}$ - Volumetric strain at radius $R$

$\varepsilon$ - Principal shear strain

$\dot{\eta}$ - Volumetric strain rate

$\dot{\varepsilon}$ - Principal shear strain rate

$\rho$ - Density of crushed material

$\bar{\rho}_{i}-\quad$ Average density of crushed material in zone $i$

$A(t)$ - Function of time, complementary solution factor for differential equation of motion 


$$
\begin{aligned}
& A_{i}-\quad \text { Values of } A(t) \text { used temporarily in derivation } \\
& B_{i}(r, t) \text { - Function of time and radius } r \text { for average } \\
& \text { density } \bar{\rho}_{i} \text {, see Equations } 56,57 \text { and } 58 . \\
& \dot{F}-\quad \text { Function of time, see Equation } 17 \\
& v-\quad \text { Time rate of change of } F \text {, see Equation } 52 . \\
& v_{R}-\quad \text { Velocity of crushed material element } \\
& \text { Velocity of crushed material element just inside } \\
& \text { the radius } R
\end{aligned}
$$

\section{Material Properties and Functions}

$$
\begin{array}{ll}
\rho_{0}- & \text { In situ density } \\
\rho_{C}- & \text { Compressed density of material under uniaxial } \\
& \text { compression strength loading } \\
\rho_{\ell}- & \text { Density of material at near solid compaction } \\
\eta_{C}- & \text { Volumetric strain under uniaxial compression } \\
\eta_{\ell}- & \text { Strength loading } \\
\sigma_{C}- & \text { Uniaxial compression strength } \\
T_{0}- & \text { Unconfined strength (stress difference) for } \\
K_{0}^{\prime}- & \text { unbroken rock } \\
K_{2}- & \text { Unconfined strength (stress difference) for } \\
p_{\ell}- & \text { Sroken rock }
\end{array}
$$




$\mu-\quad$ Slope of shear resistance (stress difference)
versus confining pressure for broken rock
a- $\quad$ Function of $\mu$, see Equation 9
b- $\quad$ Wanction of $\mu$, see Equation 55
$c-\quad$


Acknowledgements

The author expresses his gratitude to: Neil T. Davie for checking and using the equations developed in this report and for the use of his computations and charts of penetrator response, George E. Reis for his comprehensive reading of the draft report and for his constructive criticisms, Don $B$. Longcope for his critical review and suggestions, Stan D. Meyer for the use of his computations of water entry loading, and to Richard $T$. Othmer for his continuously helpful encouragement and review of the written version of this work. 
List of References

1. N. T. Davie, "A Method for Describing Nearly Normal

Penetration of an $0 \mathrm{give}$ Nosed Penetrator into Terrestrial Targets," Sandia National Laboratories Report SAND79-0721, June 1979, Albuquerque, New Mexica.

2. B, Ross and S. Hanugud, "Penetration-Studies of Ice with Application to Arctic and Subarctic Warfare," Final Report, Project NWCR 7000-452-4, (SRI Project 7000-452) September 1969, Menlo Park, California.

3. F. R. Norwood, "Cylindrical Cavity Expansion in a Locking Soil," Sandia National Laboratories Report SLA-74-0201, July 1974, Albuquerque, New Mexico.

4. P. Yarrington, "A One-Dimensional Approximate Technique for Earth Penetration Calculations," Sandia National Laboratories Report SAND77-1126, September 1977, Albuquerque, New Mexico.

5. D. Z. Yankelevsky and J. Gluck, "Nose Shape Effect on High Velocity Soil Penetration," Int. J. of Mech. Sci., Vol. 22, pp 297-311, 1980 .

6. S. Hanagud and B. Ross, "Large Deformation, Deep Penetration Theory for a Compressible Strain-Hardening Target Material, "AIAA J., Vol. 9, No. 5, May 1971.

7. M. J. Forrestal, D. B. Longcope, and F. R. Norwood, "A Model to Estimate Forces on Conical Penetrators into ory Porous Rock," J. of Applied Mechanics, Vol. 48, March 1981.

8. C. H. Yew and P. P. Stirbis, "Penetration of Projectiles into Terrestrial Target," J. Eng. Mech. Div., ASCE 104, EM3, 1978.

9. N. T. Davie, report to be written, Sandia National Laboratories, Albuquerque, New Mexico.

10. S. D. Meyer, unpublished computations, Sandia National Laboratories, Albuquerque, New Mexico, July 1981.

11. Mathematical Studies Relating to Military Physical Research," Summary Technical Report of the Applied Mathematics Pane 1, NDRC, NDRC-STK-AMP-1, Vol. 1, Washington, D.C., 1946, Declassified November 23, 1960. 
12. "The Force of Impact on a Sphere Striking a Water Surface" (Second Approximation), Unclassified AMP Report 42.2R, OEM sr-945, OSRD 5485, AMG-NYU, Prepared by the Applied Mathematics Group, New York University, July 1945.

13. C. H. Corley, "Laboratory Tests on Antelope Tuff for Rock Penetration Studies," Terra Tek, Salt Lake City, Utah, to be published.

14. M. M. Hightower, informal communications, Sandia National Laboratories, Albuquerque, New Mexico, February, 1981. 
Distribution:

Director

Defense Nuclear Agency

Attn: SPSS

Washington, D. C. 20305

For: E. Sevin

Lawrence Livermore National Laboratory

P. 0. Box 808

Livermore, CA 94550

Attn: G. R. Goudreau, Technical Library

Avco Corporation

Government Products Group

201 Lowe 11 Street

Wilmington, MA 01887

Attn: D. Henderson

California Research and Technology, Inc.

6269 Variel Avenue

Woodland Hills, CA 91364

Attn: K. N. Kreyenhagen

Waterways Experimental Station

Soil Dynamics Division

Vicksburg, MS 39180

Attn: R. S. Bernard

Commander

U. S. Army Missile Command

Attn: DRC-PM-PE-EA (J.R. Pemberton)

Redstone Arsenal, AL 35802

Los Alamos National Laboratory

P. 0 . Box 1663

Los Alamos, NM 87545

Attn: Reports Library

Pacific Technology

P. 0. Box 148

Del Mar, CA 92014

Attn: R. L. Bjork

The University of Texas (2)

Department of Aerospace Engineering and

Engineering Mechanics

Austin, TX 78712

Attn: C. H. Yew

E. A. Ripperger

Army Cold Region Research and Engineering Laboratory

P. 0 . Box 282

Hanover, $\mathrm{NH} 03755$

Attn: Dennis Farrell 


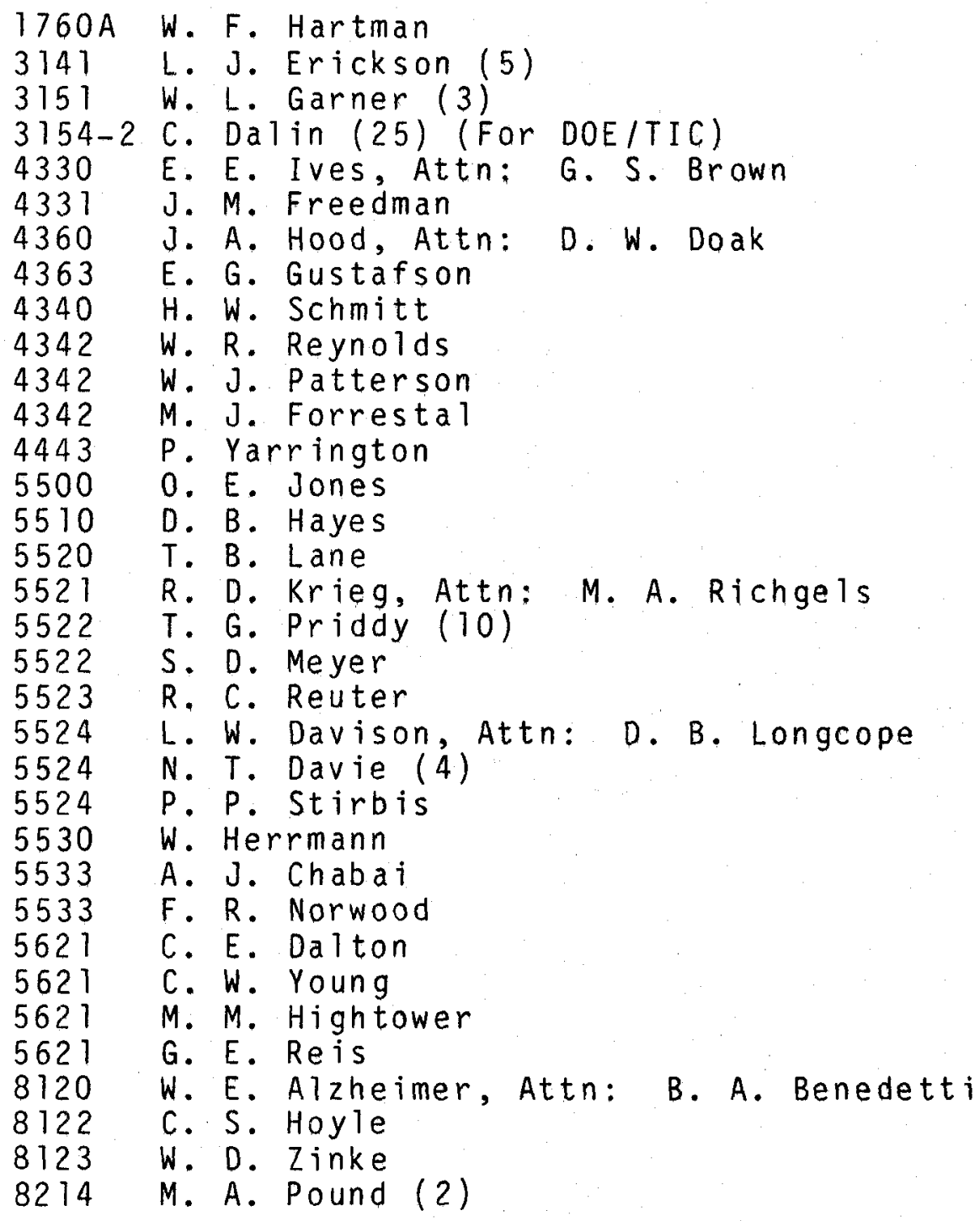

